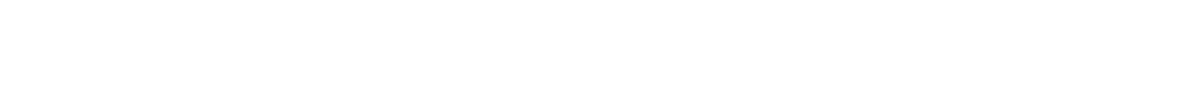

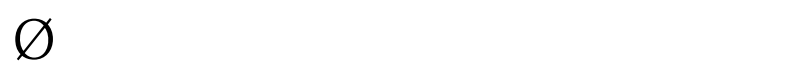

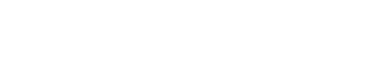

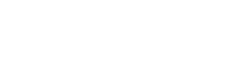

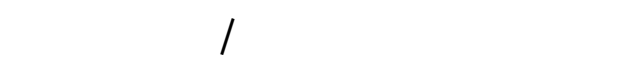

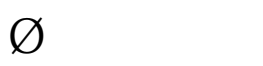

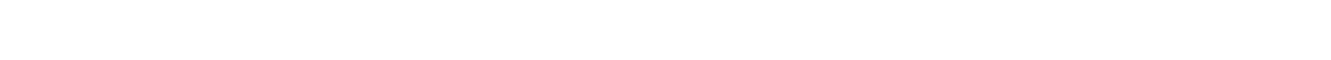

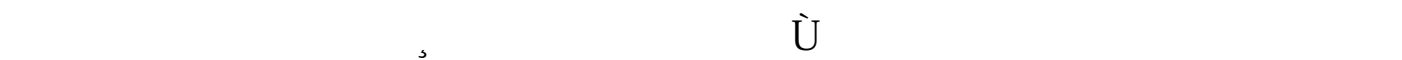

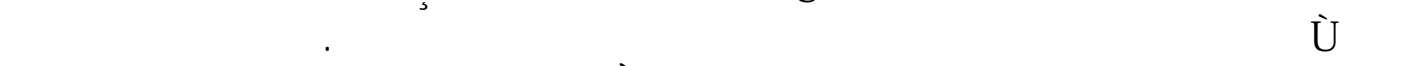

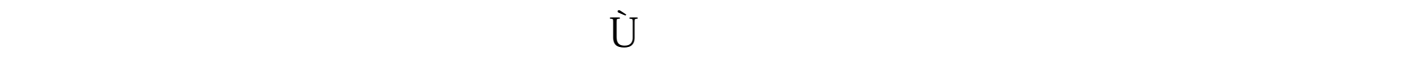

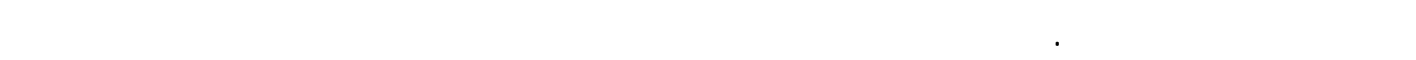

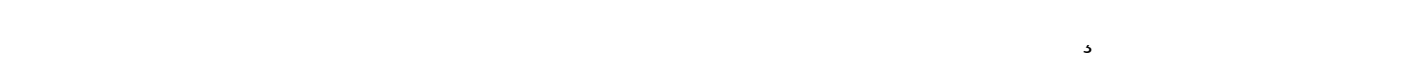

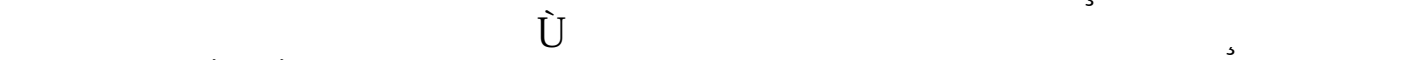

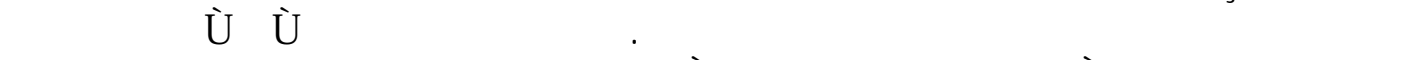
ry

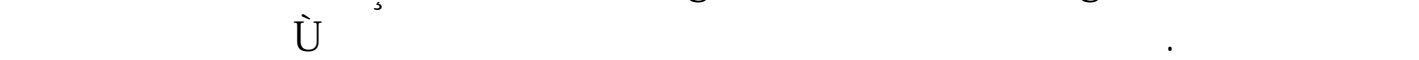

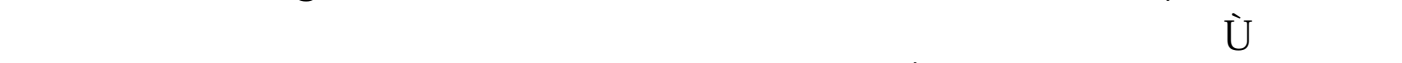

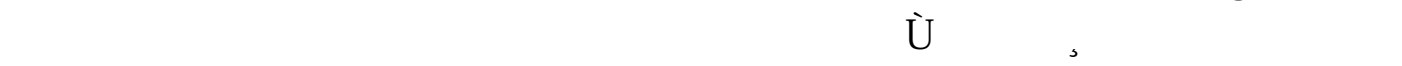

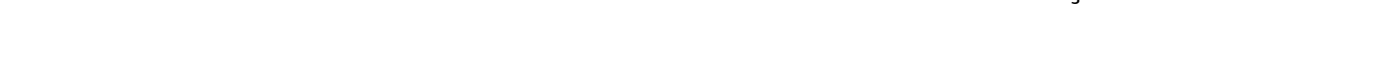

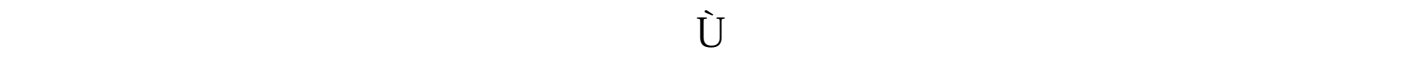

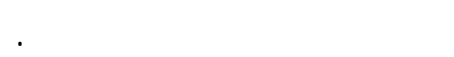

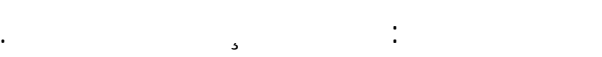

\section{Patient Privacy in Environmental Context of General Hospitals , Assessment Local Reality}

\section{Ghada mohammed yonis}

\section{Assistant lecturer}

\section{Abstract:}

The Environmental Context of General Hospital is one of The Composite and Complex Physical Environments, Which Integrate and Combine Multifunction and Diverse forms of Human Behavior, Patient Privacy as Concept, Determined by Surrounding Social / Physical Therapeutic Environment .

This Article Represent The Effects Both of Social Environment Which Interface Patient and Physical Characteristics of Therapeutic Environments, by Testing Three Types of Wards in AL-SALAM General Hospital in Mosul, The Results Indicates The Manipulation of Physical Environment to Get Accepted Level of Patient Privacy Dose not Works Without Consider Social Interaction Between Patients Together, Medical Staff and Visitors Where Those Have Affects in Patient Assessment of Privacy Level.

Key words: privacy, general Hospital 


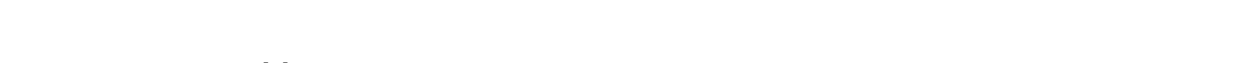

irPtore

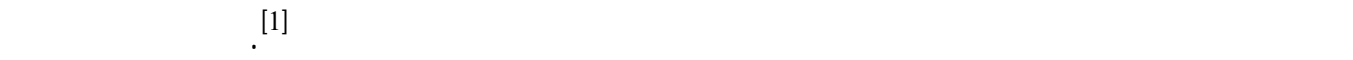

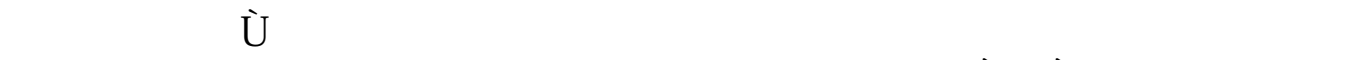
ry

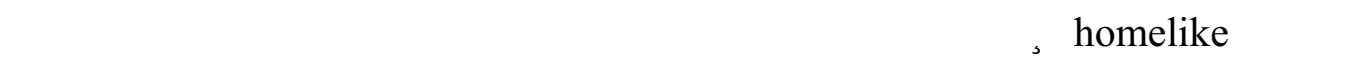

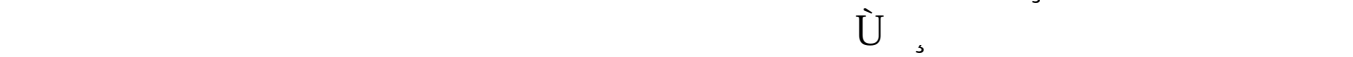

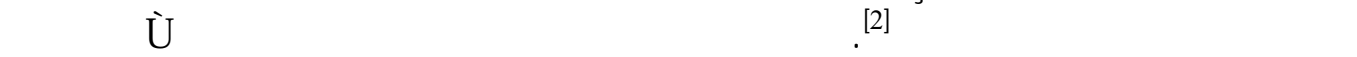
ĐX

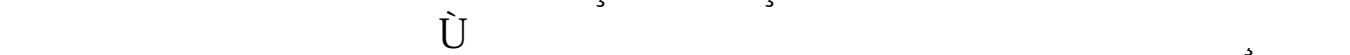

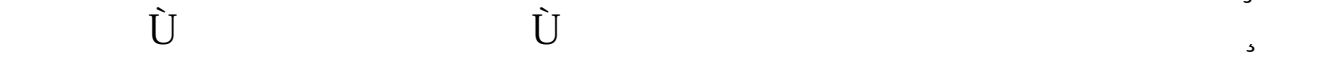

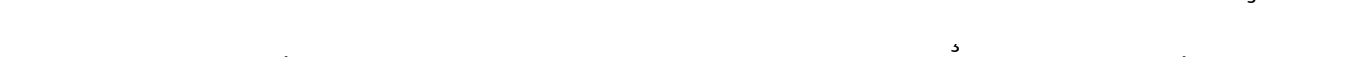

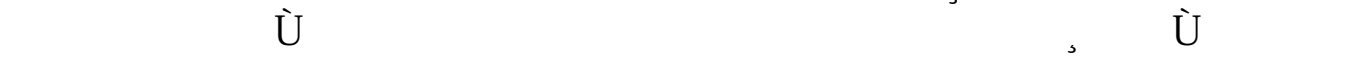

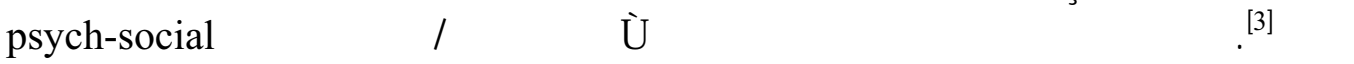

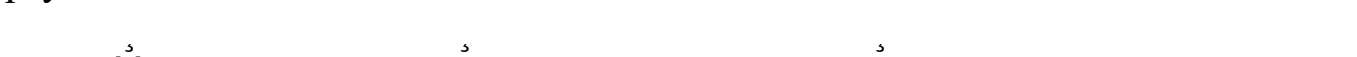

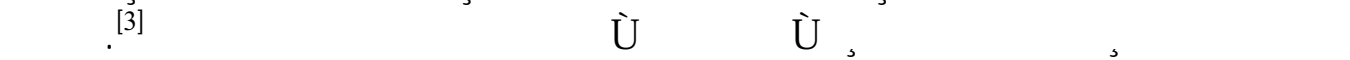

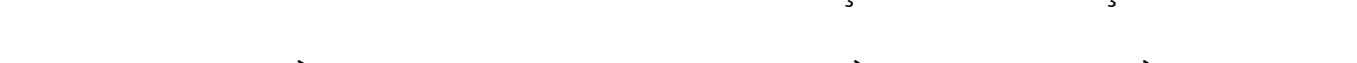

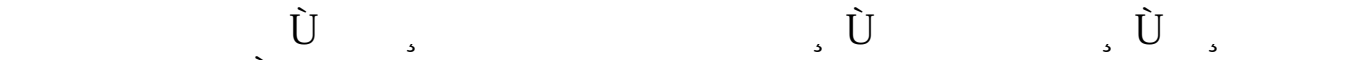

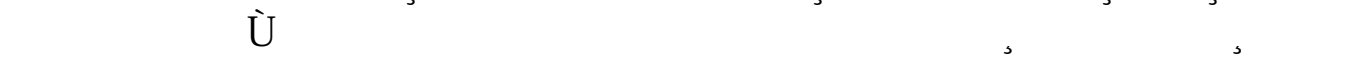

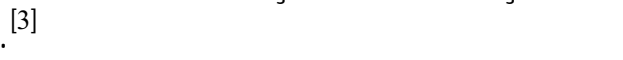

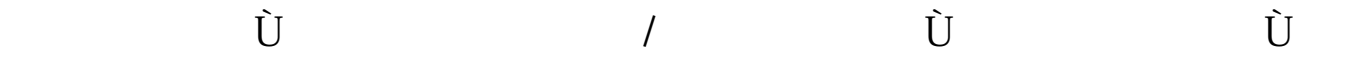

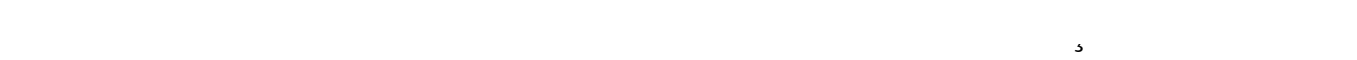
GZ̃ ŴESD Ŷ̃

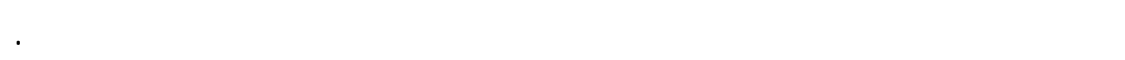

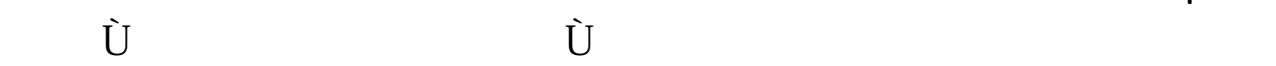

:Ŝţ nth́Ž̌ Ło

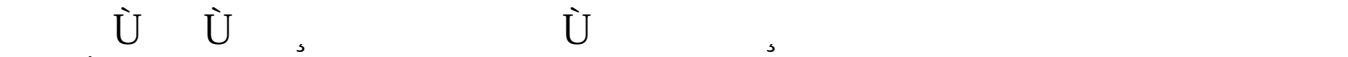

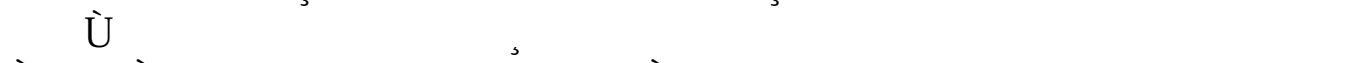

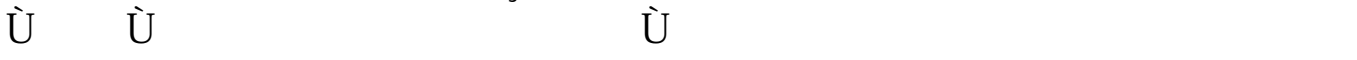

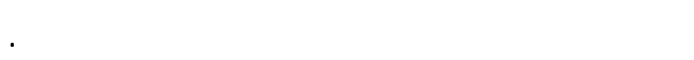

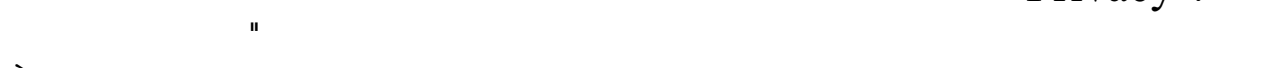

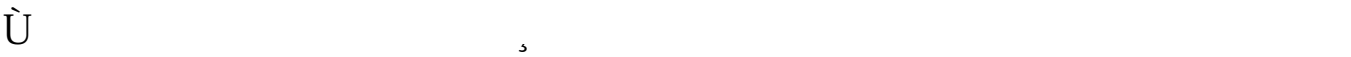

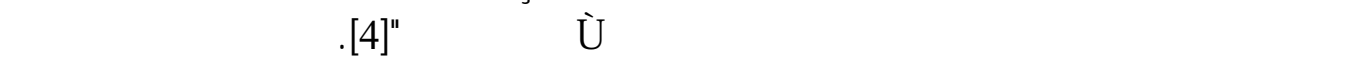

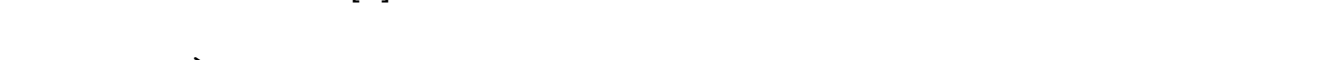

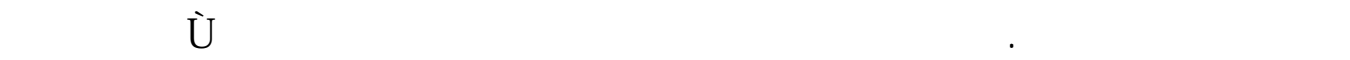

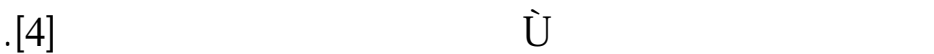

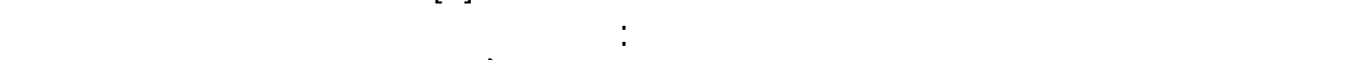

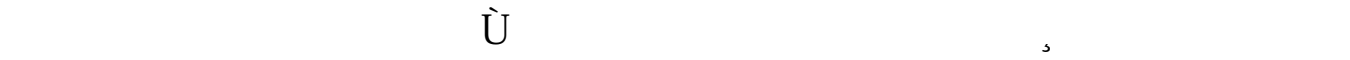

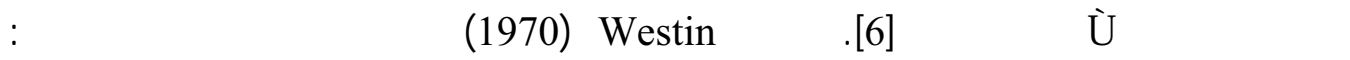

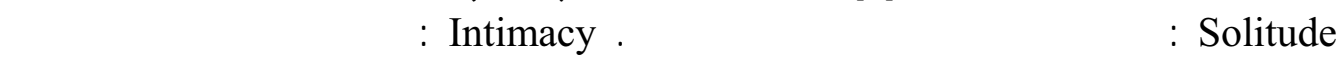




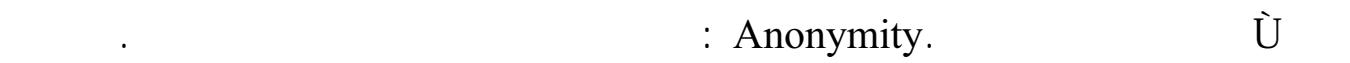

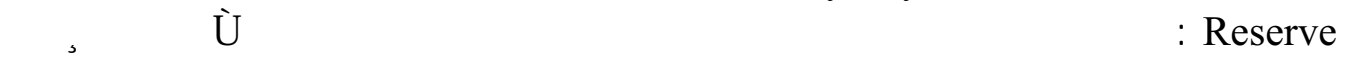

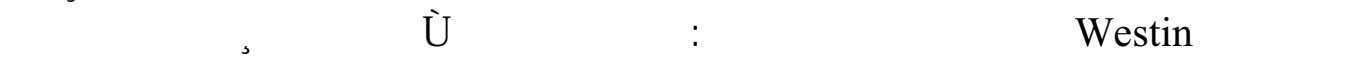

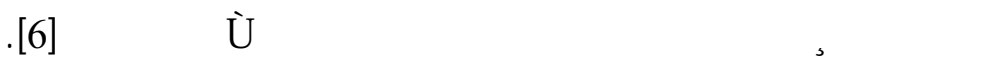

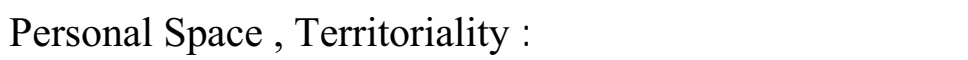

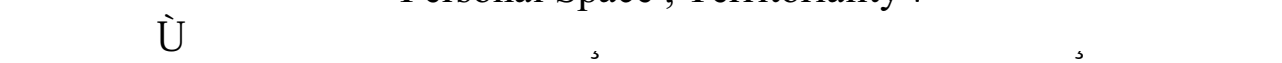

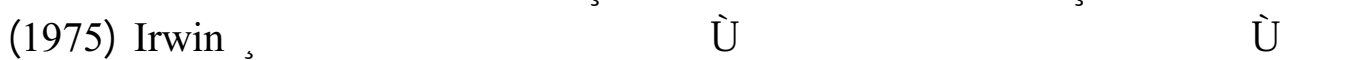

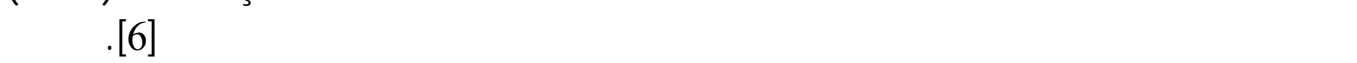

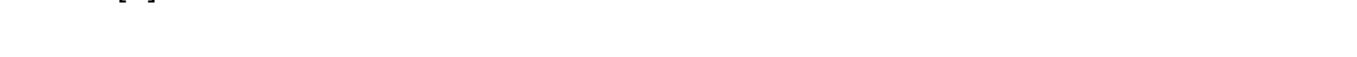

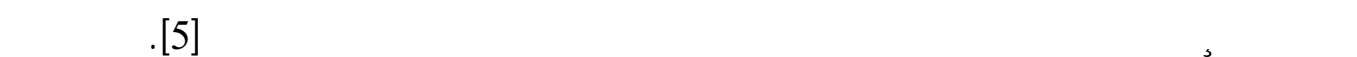

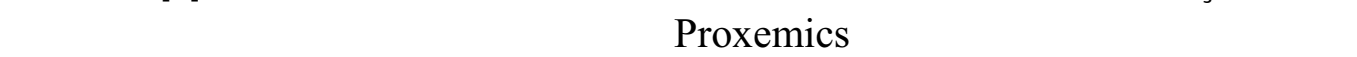

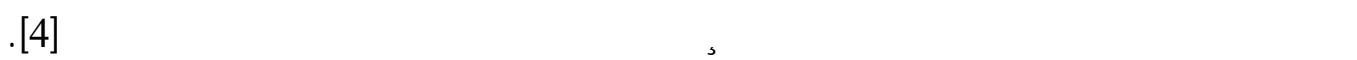

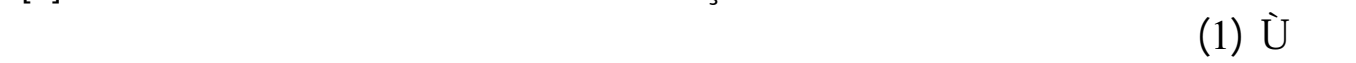

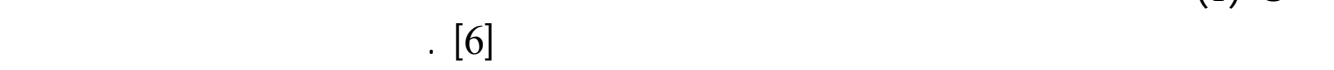

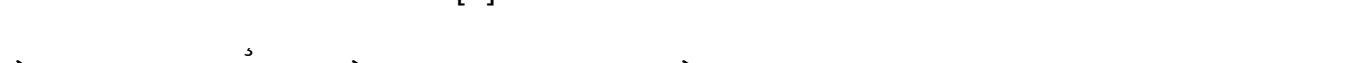

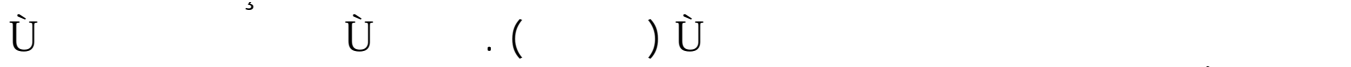

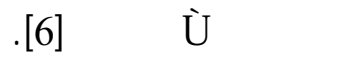

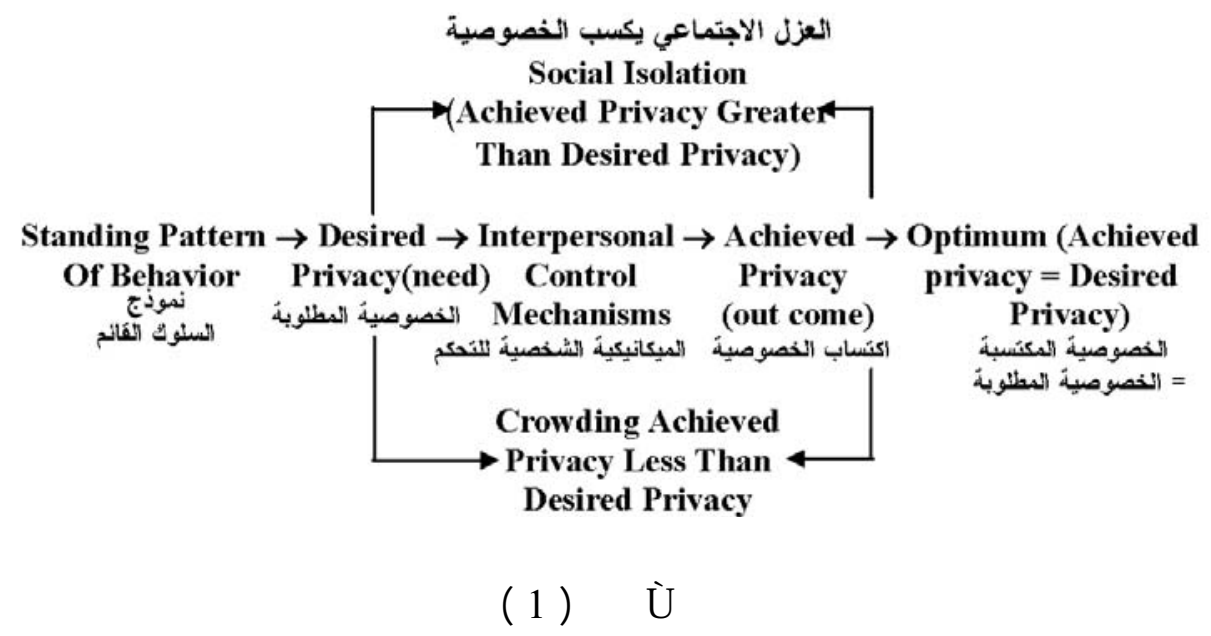

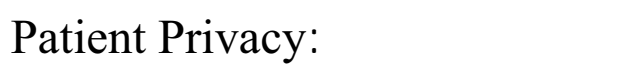

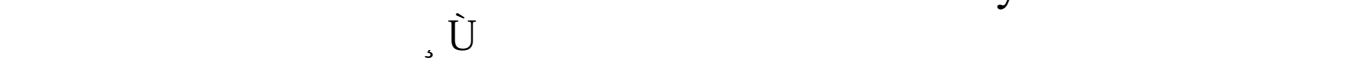

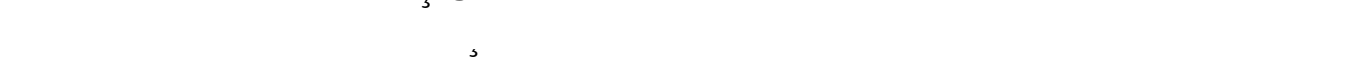

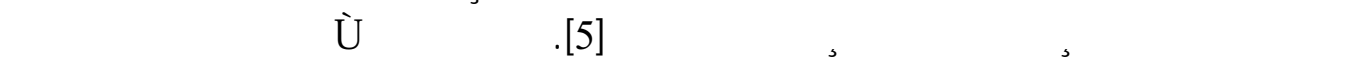

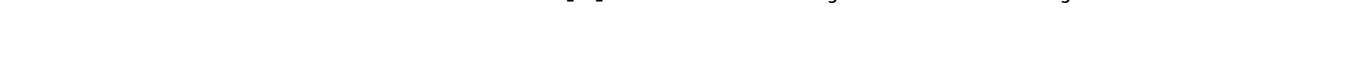

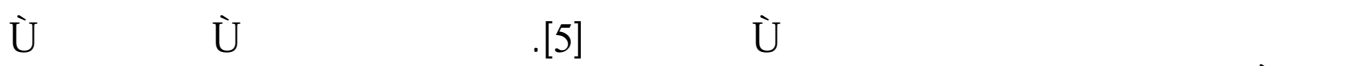
Đy

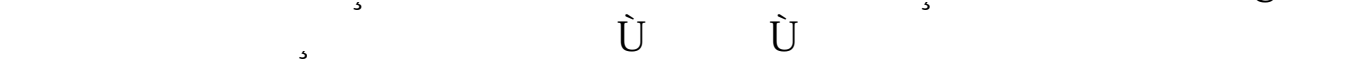

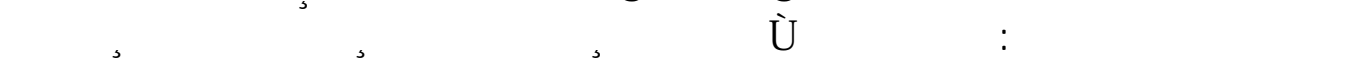

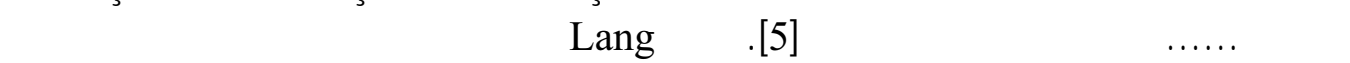

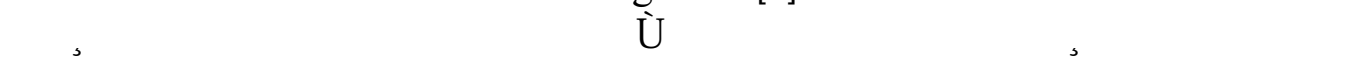




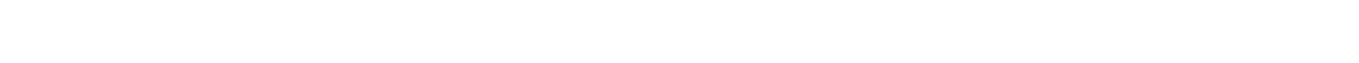

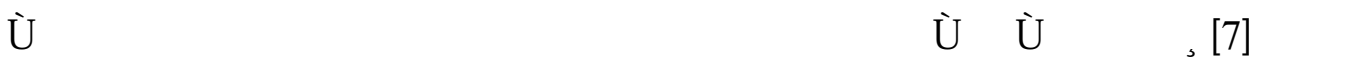

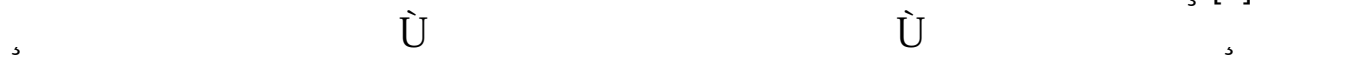

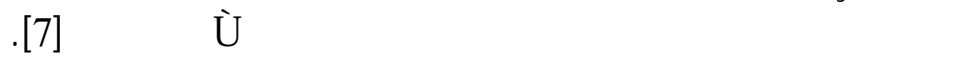

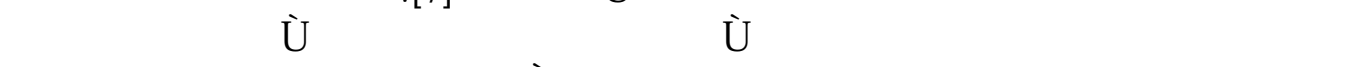

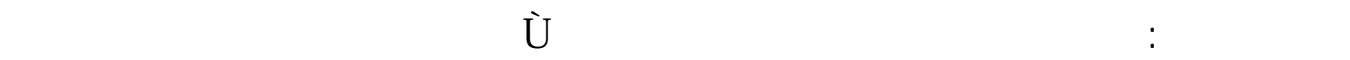

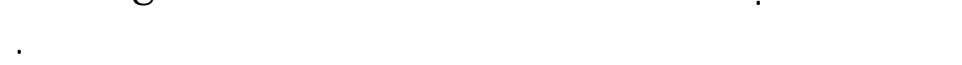

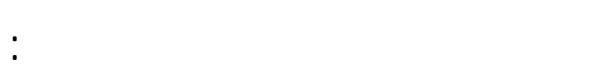

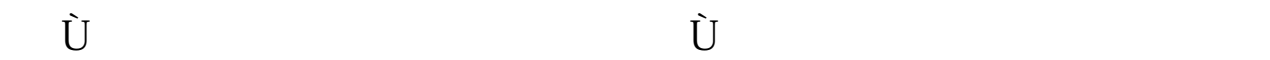

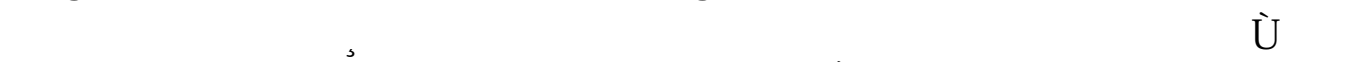

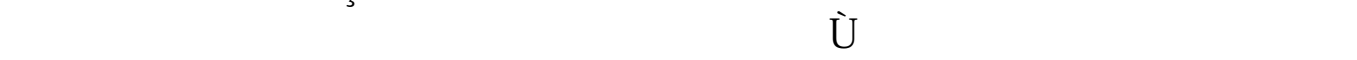

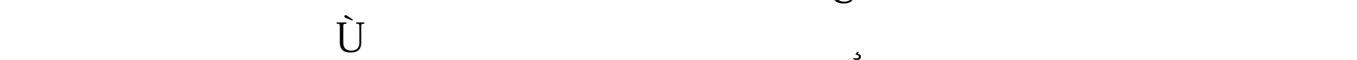

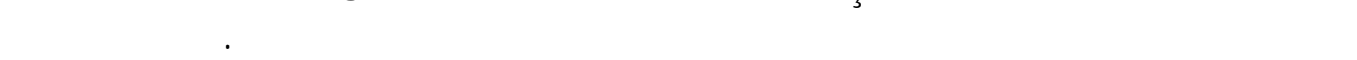

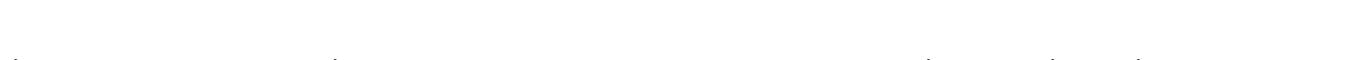

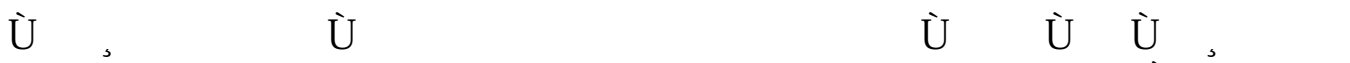

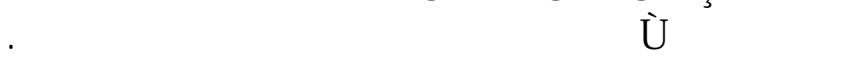

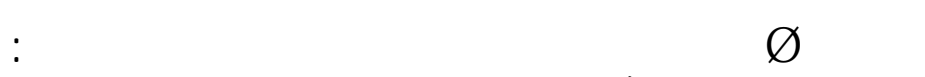

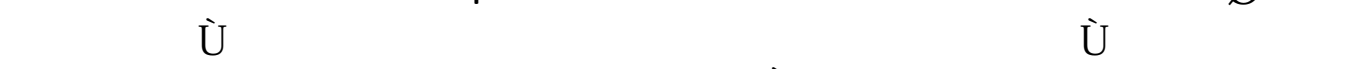

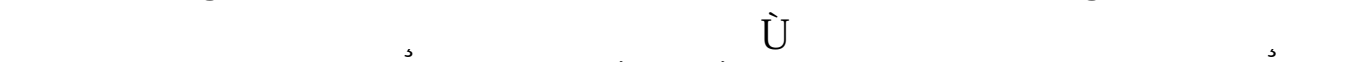

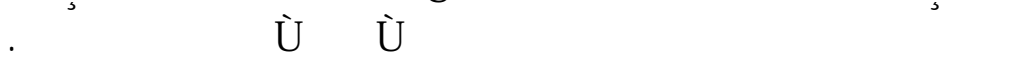

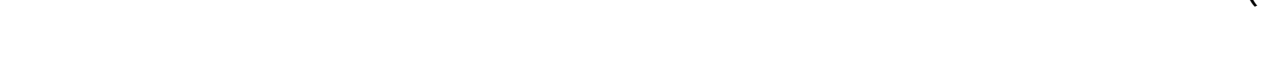

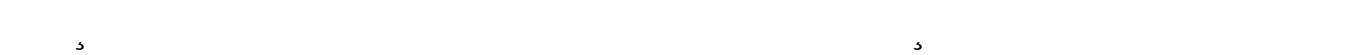

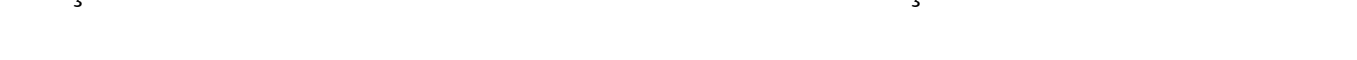

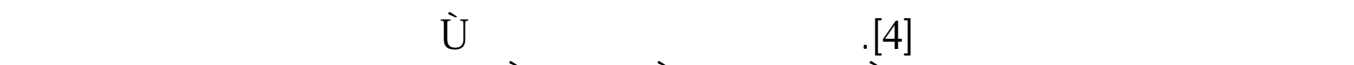

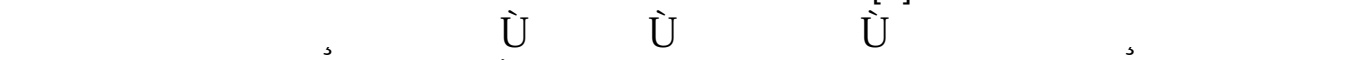

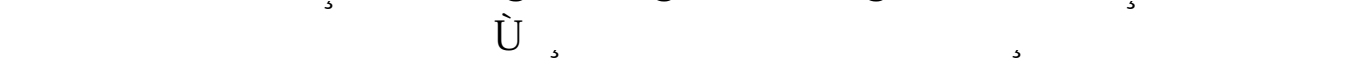

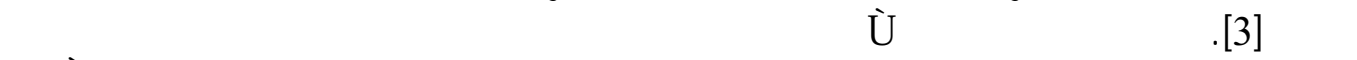
ĐC ¿ Y̌šś

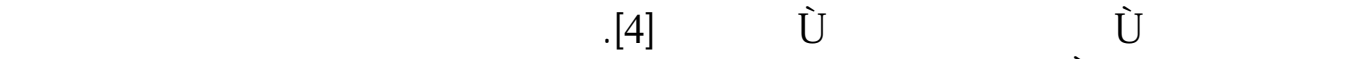

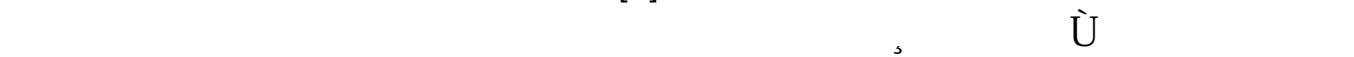

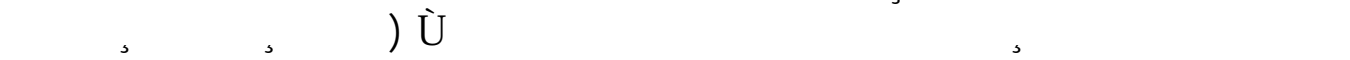

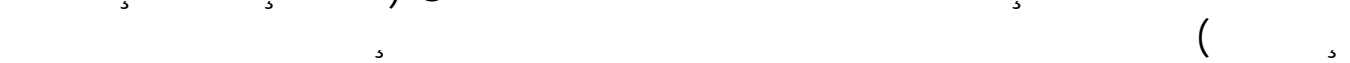

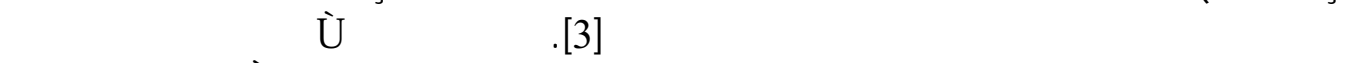

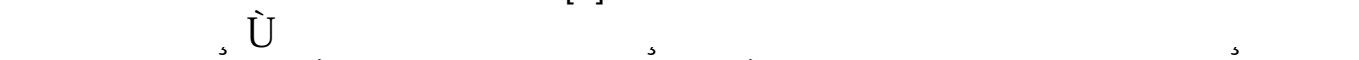

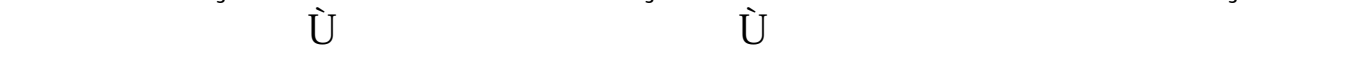

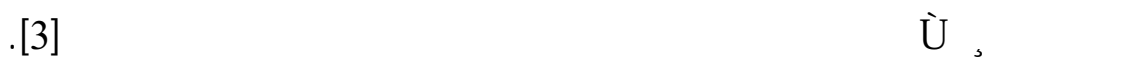

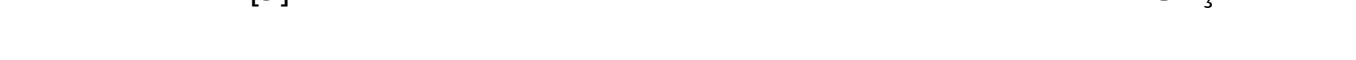

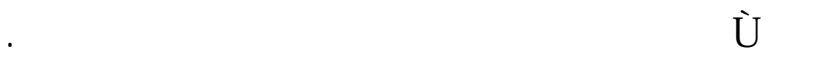




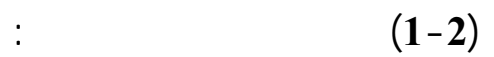

पŚ

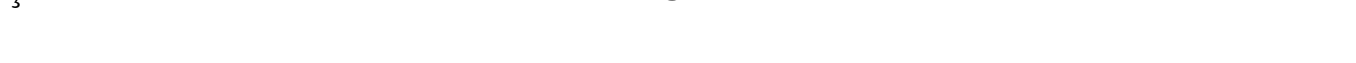

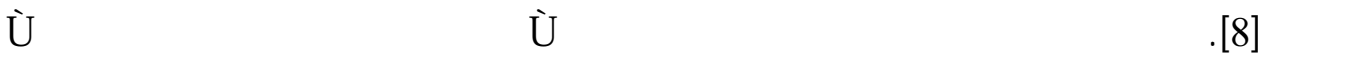

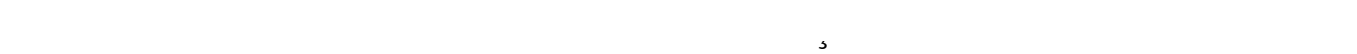

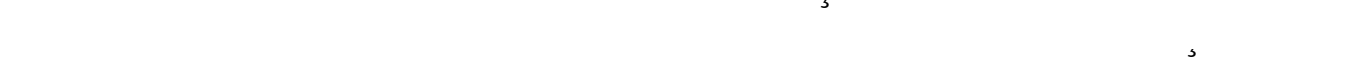
.[8]గ̌⿱宀⿻三丨

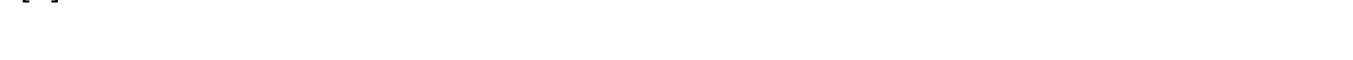

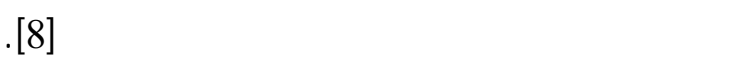

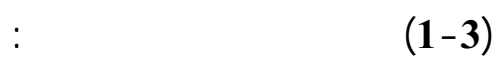

r゙ด̋

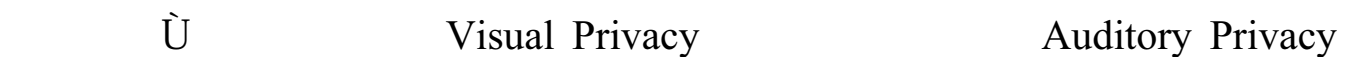

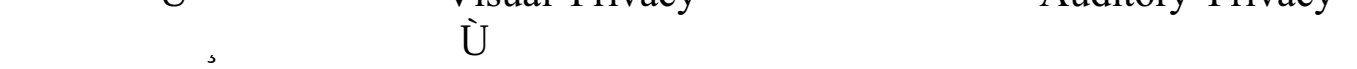

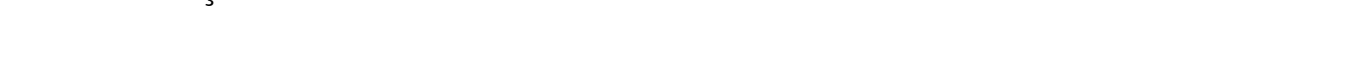

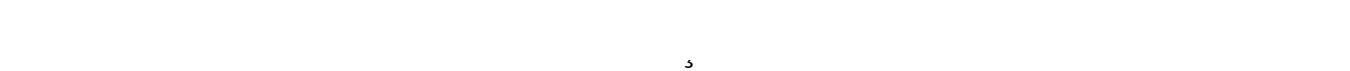

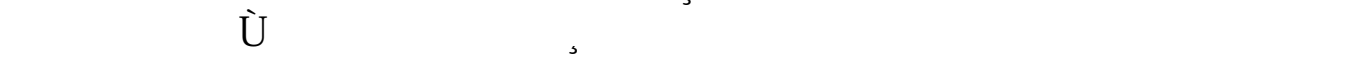

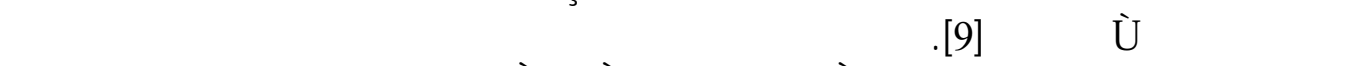

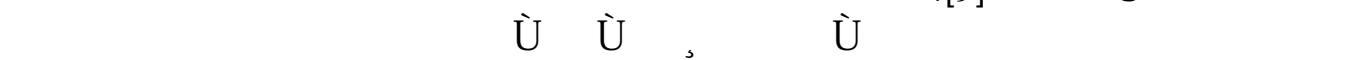

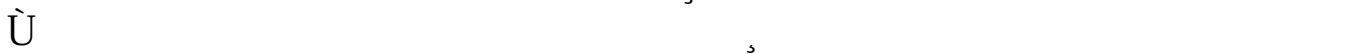

.[9]Ś ŔicdŔ: UF ü YuidŔ

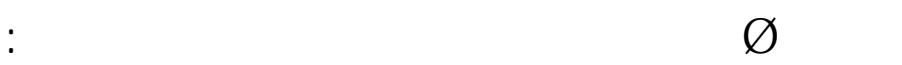

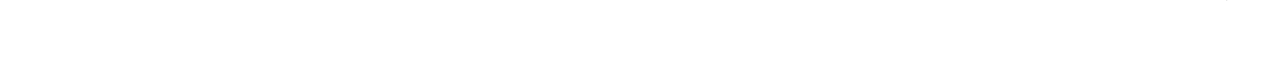
F侻 ř

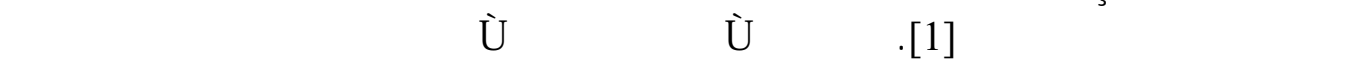

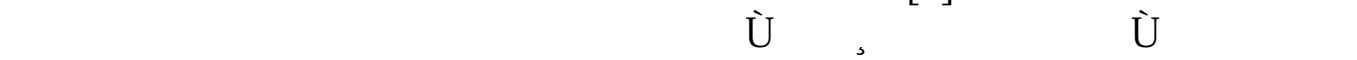

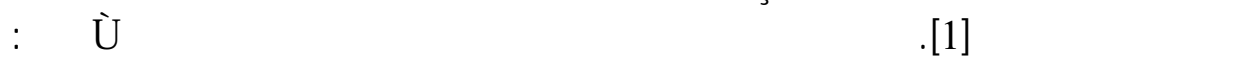

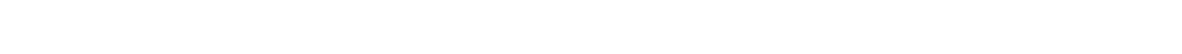

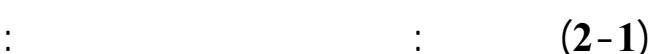

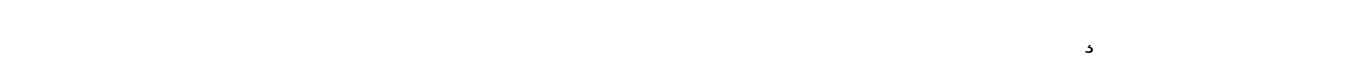

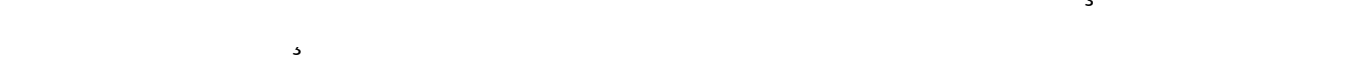

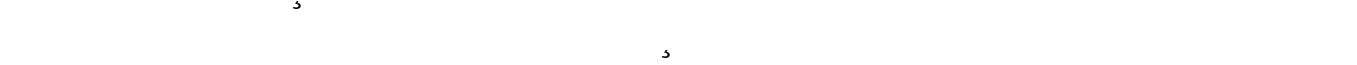

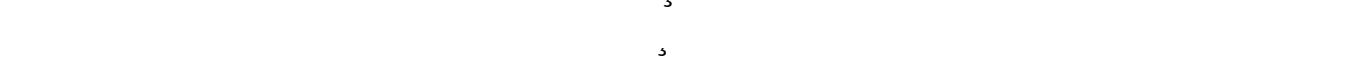

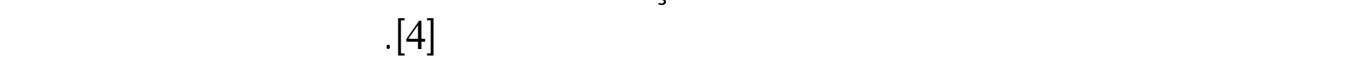

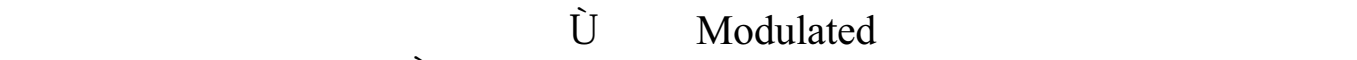

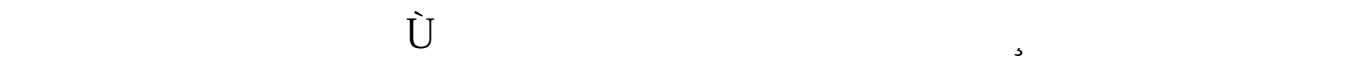

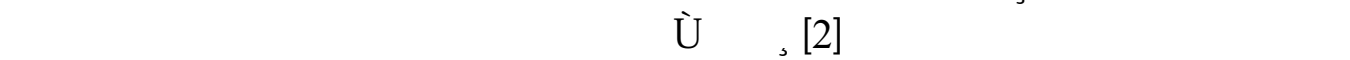

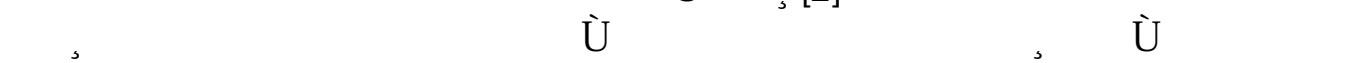

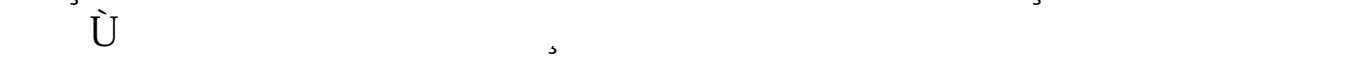

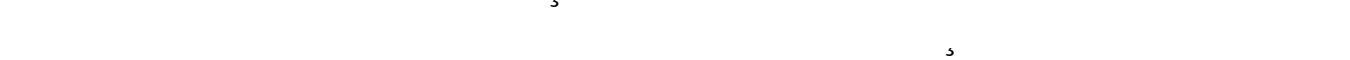




\begin{tabular}{llll} 
Al-Rafidain Engineering & Vol.14 & No.2 & 2006 \\
\hline
\end{tabular}

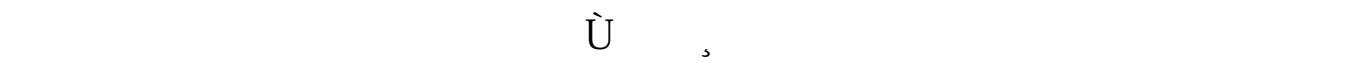

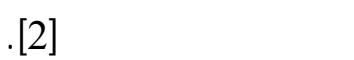

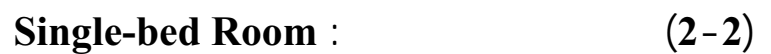

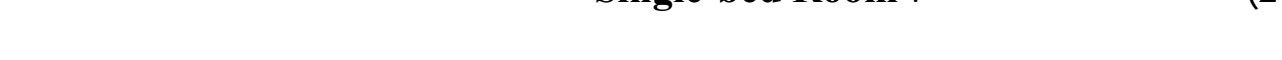

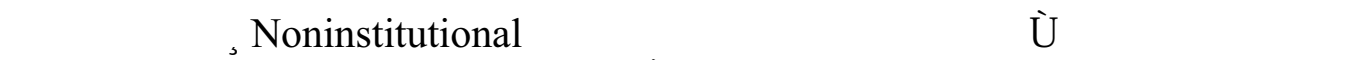

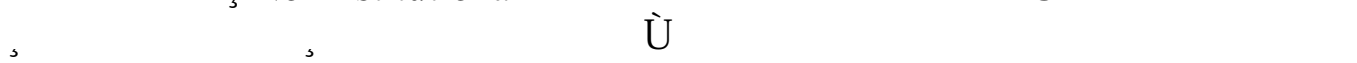

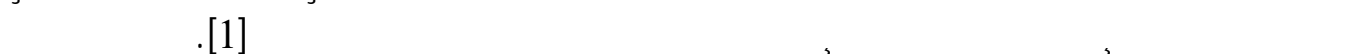

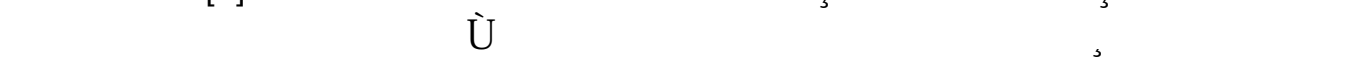

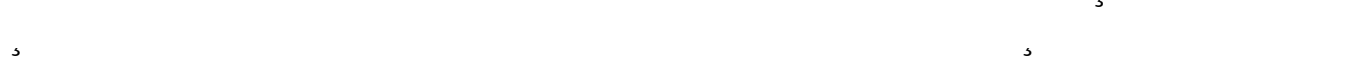

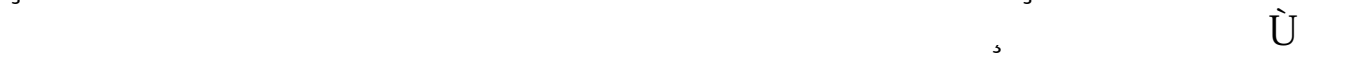

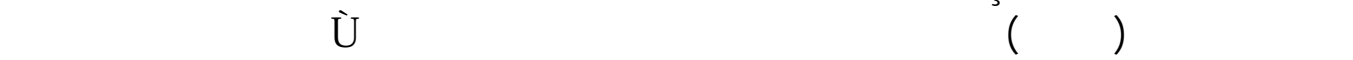

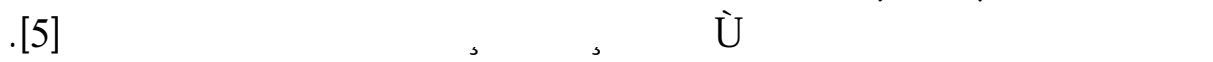

\section{:Fâ̄nofự $g$ Vịgr (2-3)}

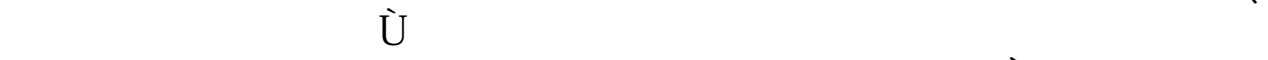
ř

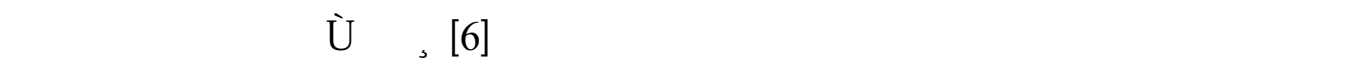

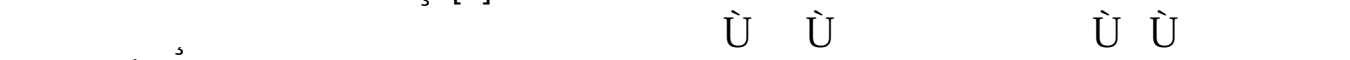

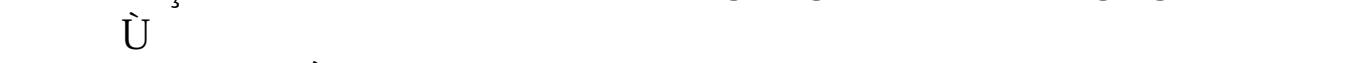

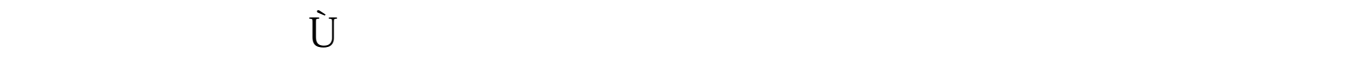

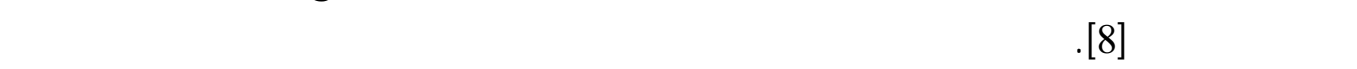

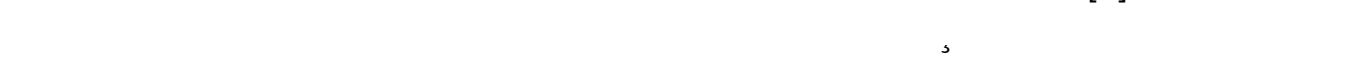

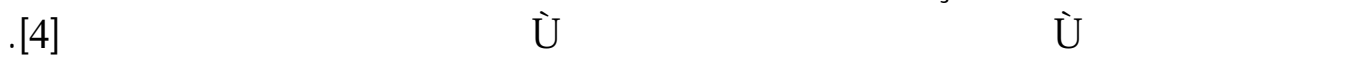

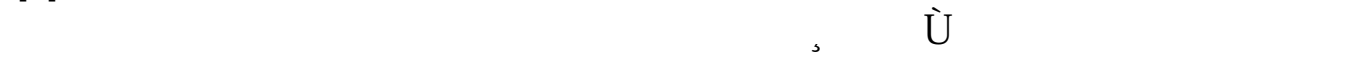

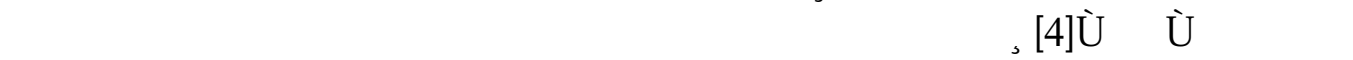

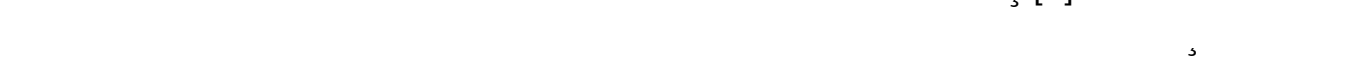

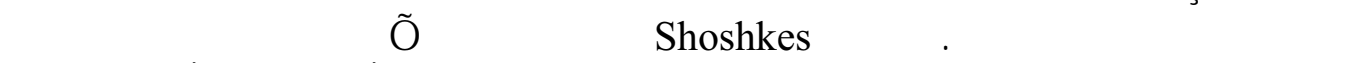

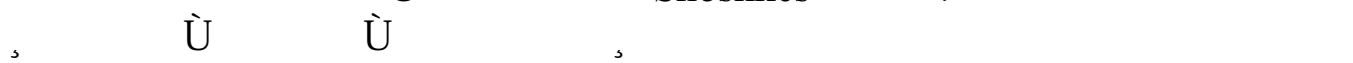

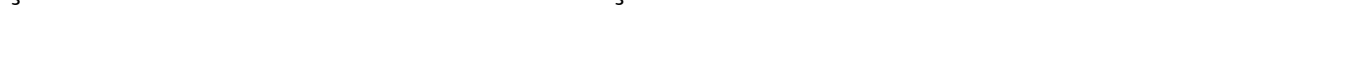
ũ

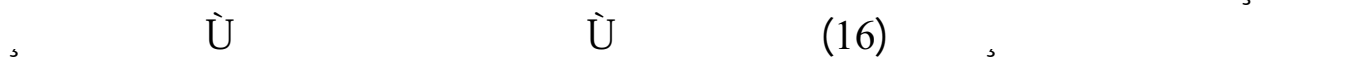

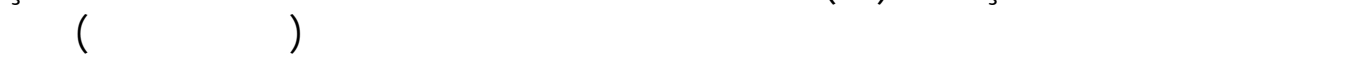

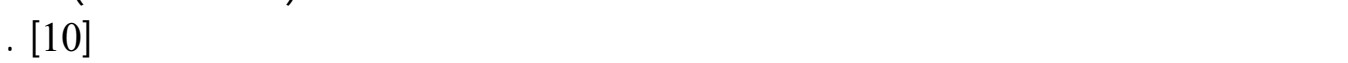

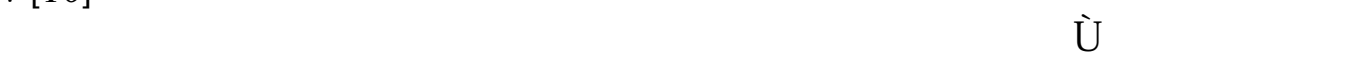

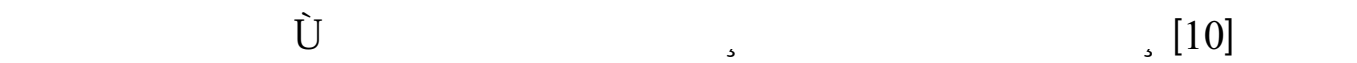

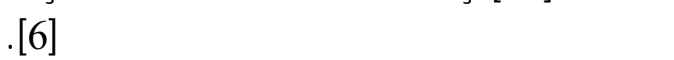

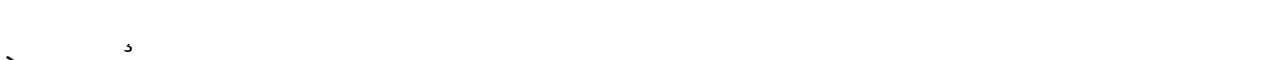

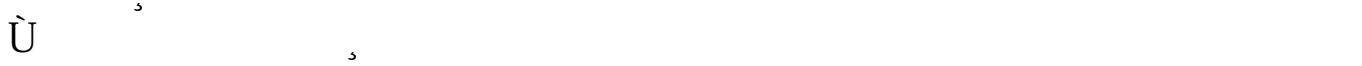




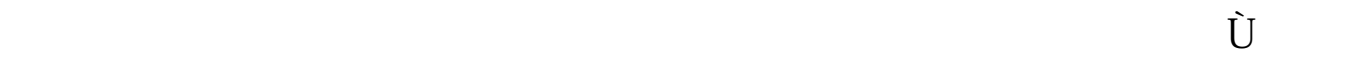

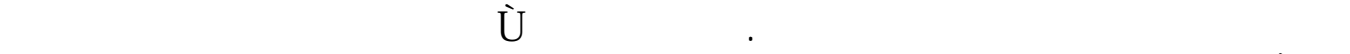

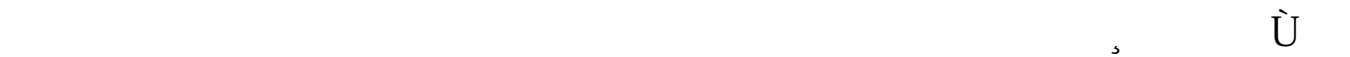

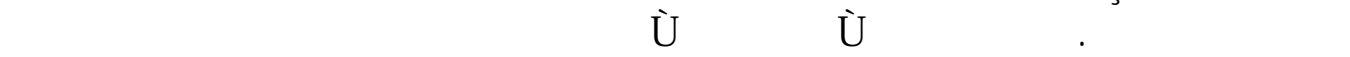

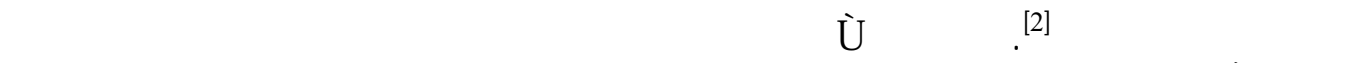

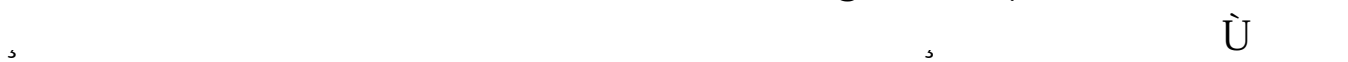

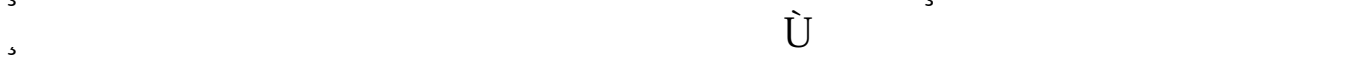

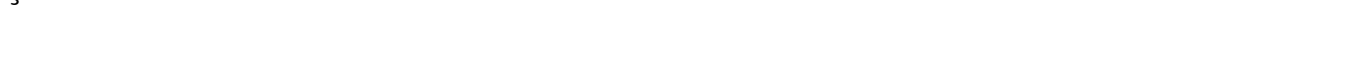

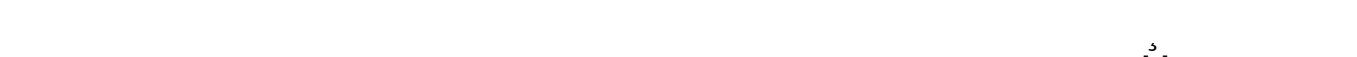
[2]

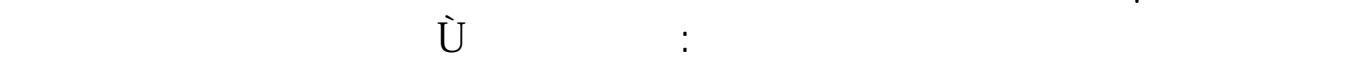

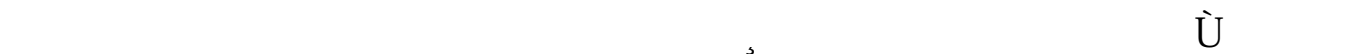

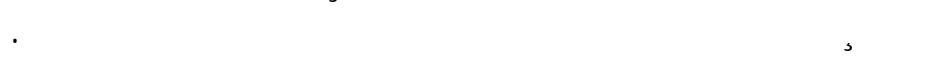

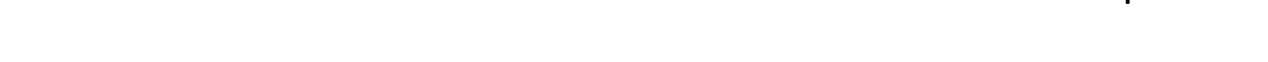

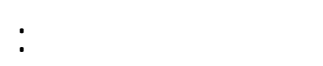

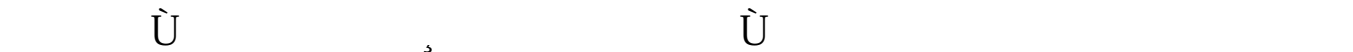

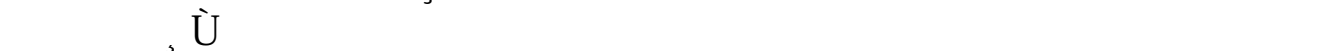
FŨ ũdŔk

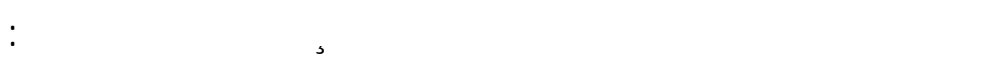

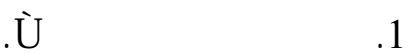

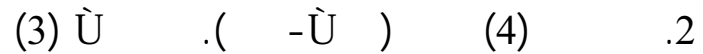

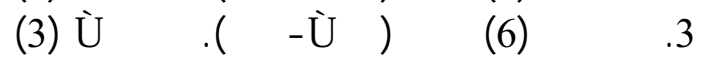

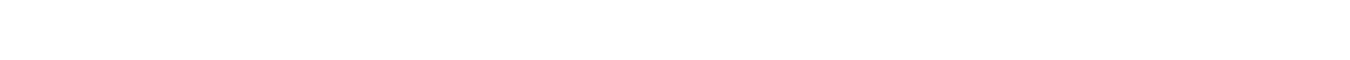

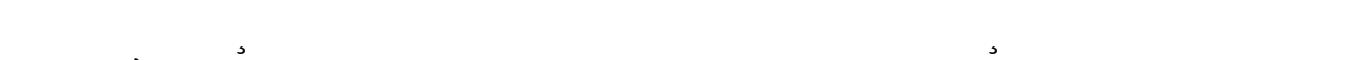

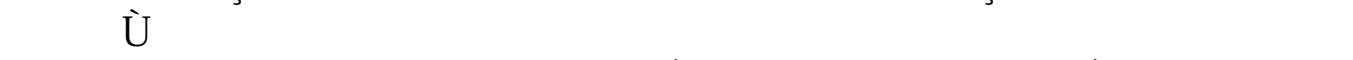

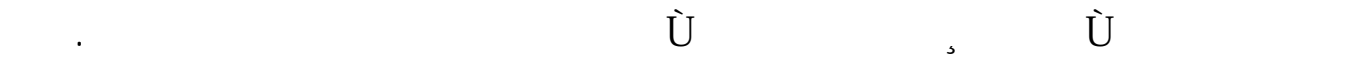

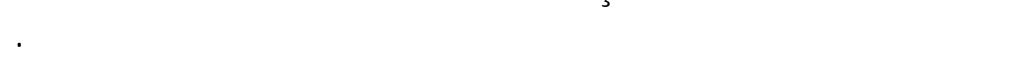




\begin{tabular}{llll} 
Al-Rafidain Engineering & Vol.14 & No.2 & 2006 \\
\hline
\end{tabular}

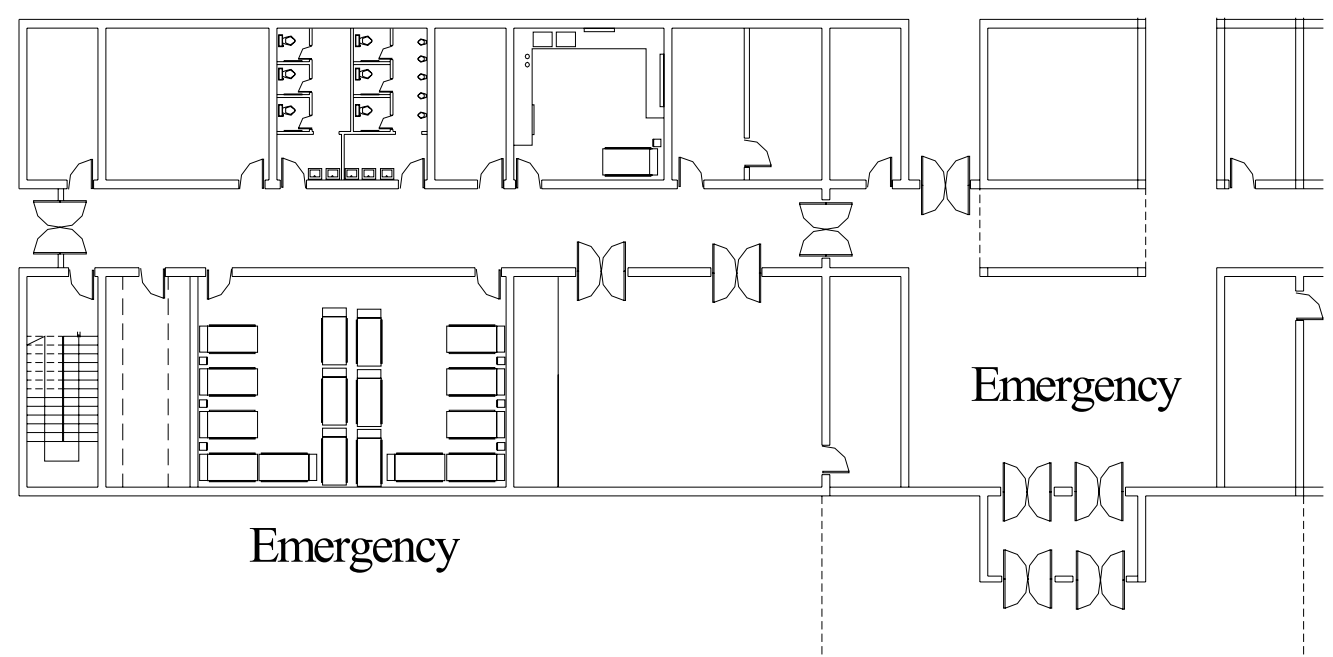

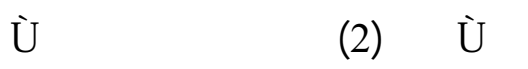

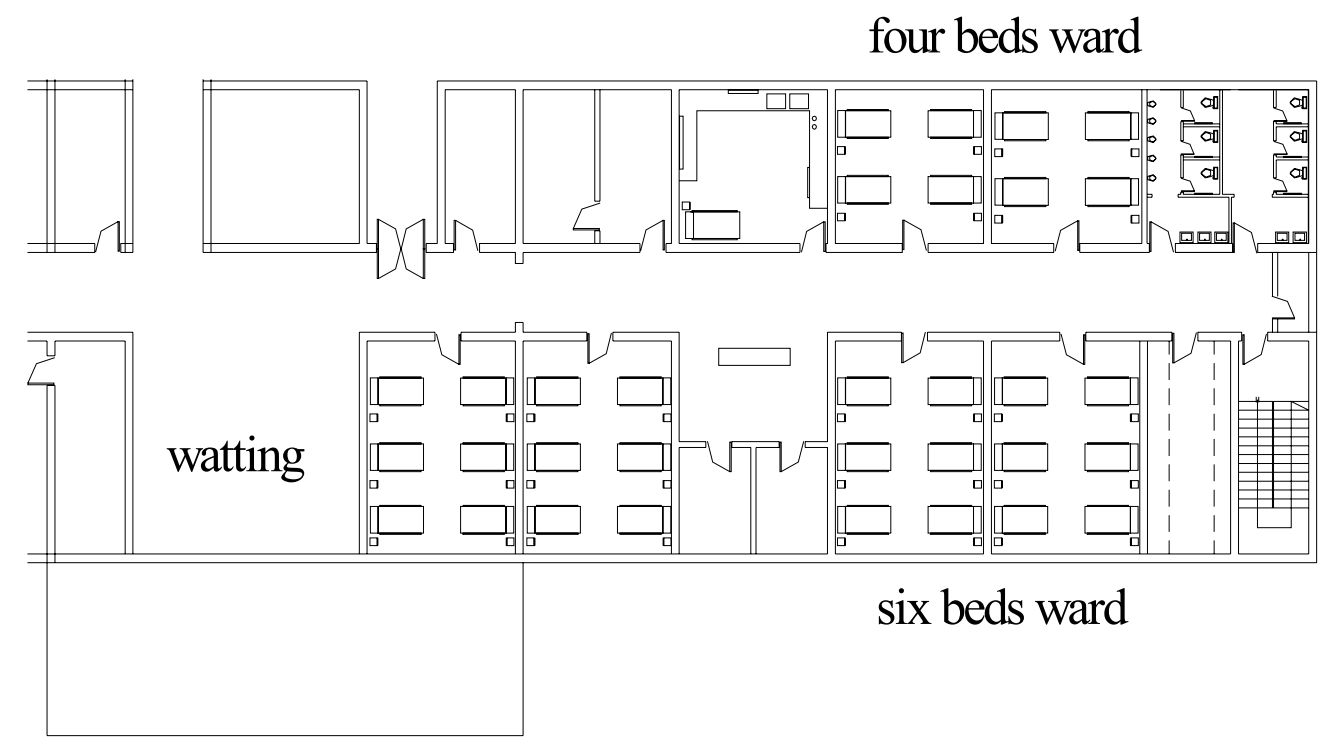

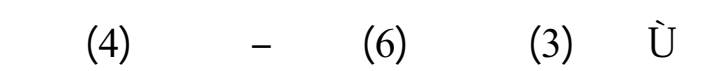




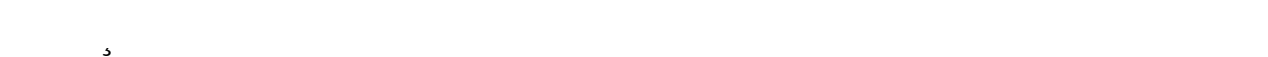
:Śšín:ịa

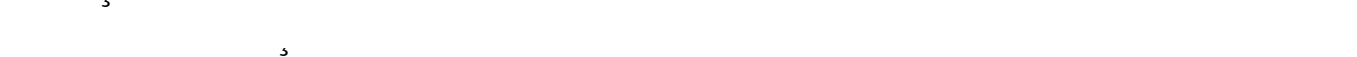

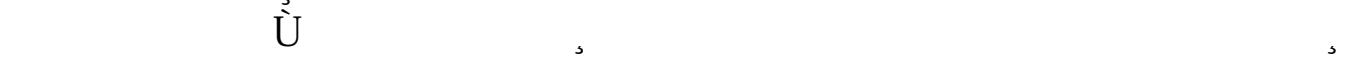

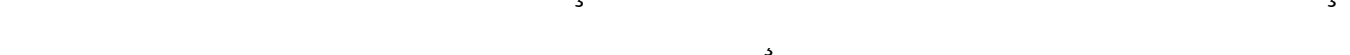

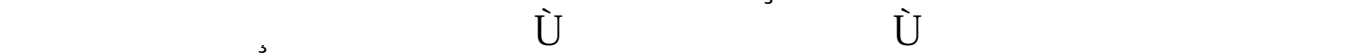

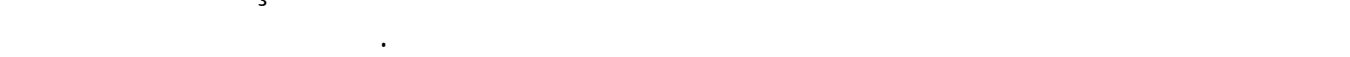

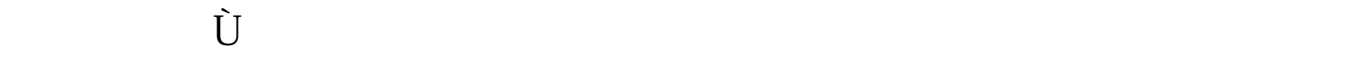

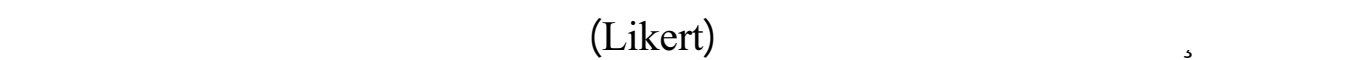

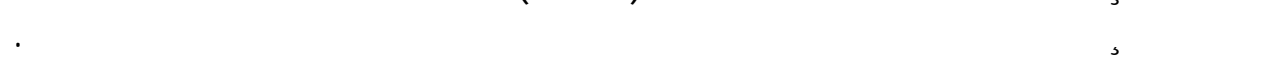

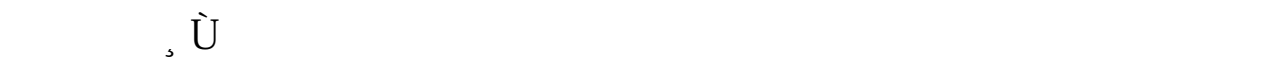
:Śšrț̣̣̂̂ি

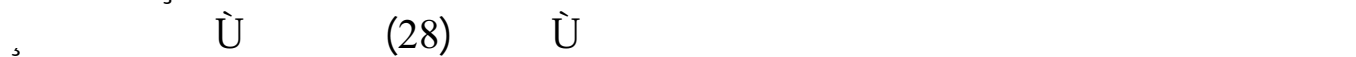

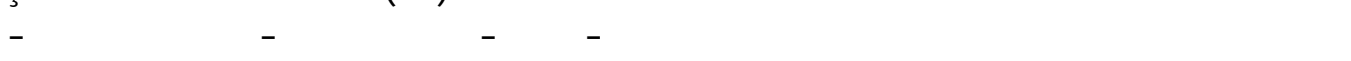

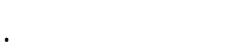

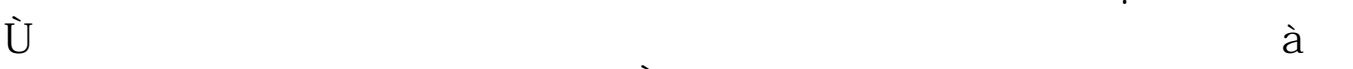

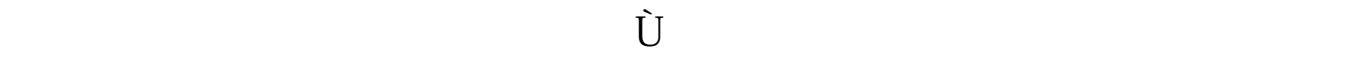

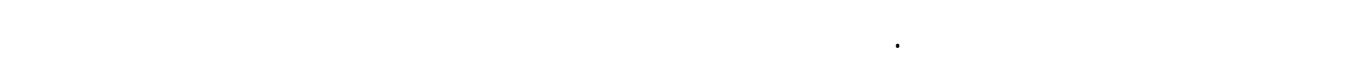

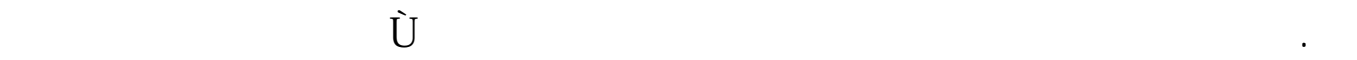

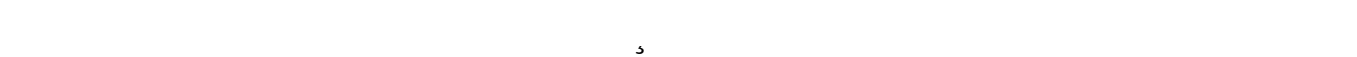

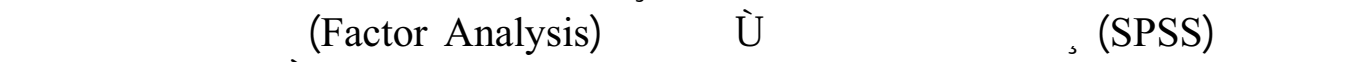

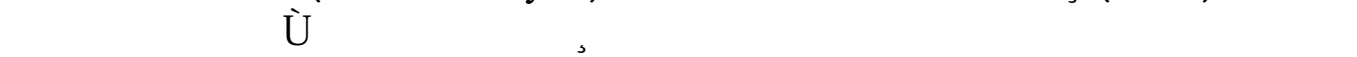

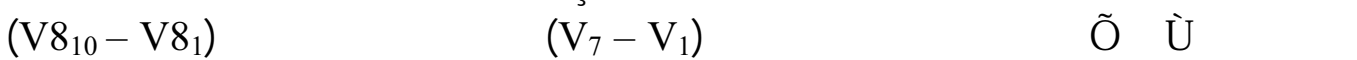

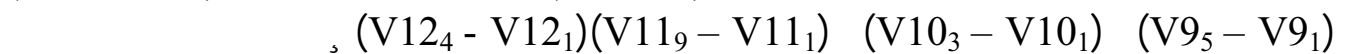

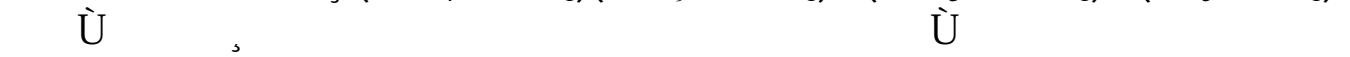

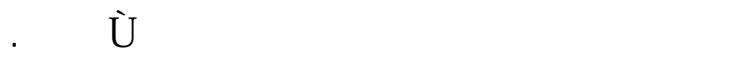

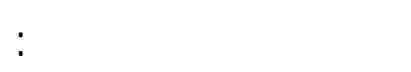

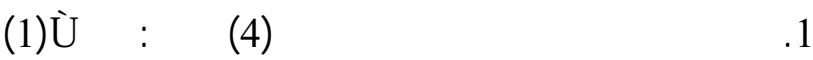

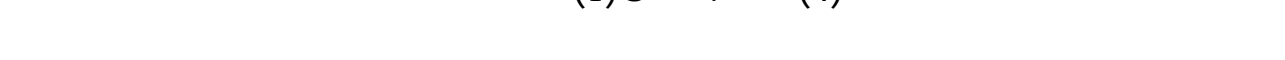

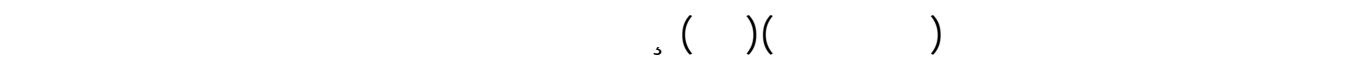

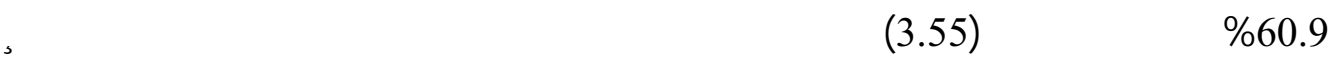

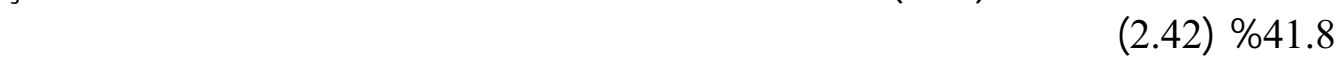
¿Ü

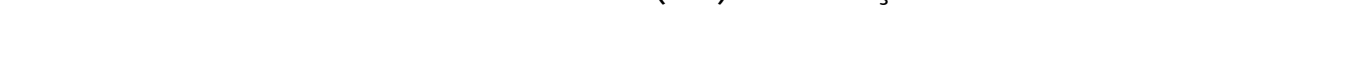

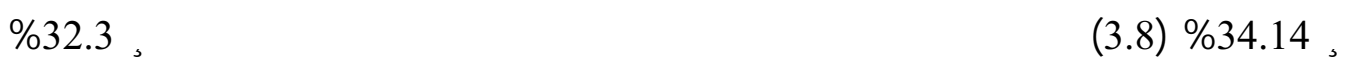

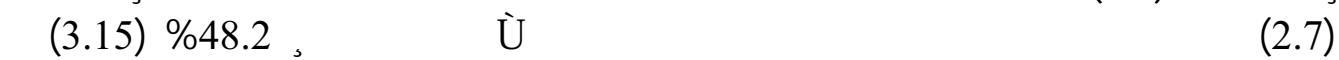

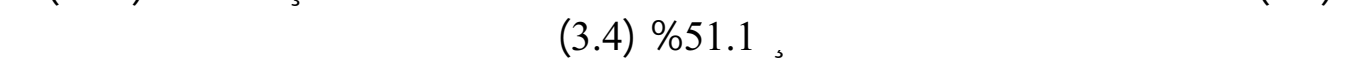

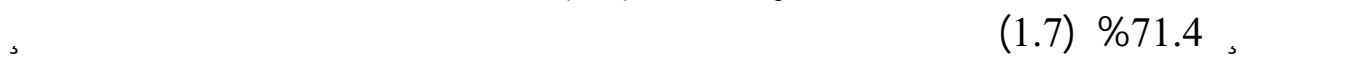

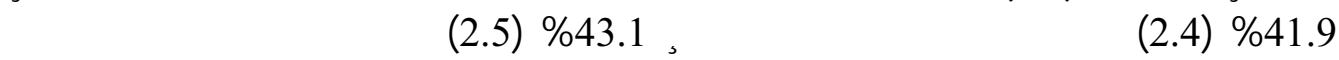

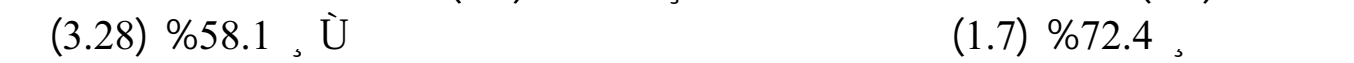




\begin{tabular}{llll} 
Al-Rafidain Engineering & Vol.14 & No.2 & 2006 \\
\hline
\end{tabular}

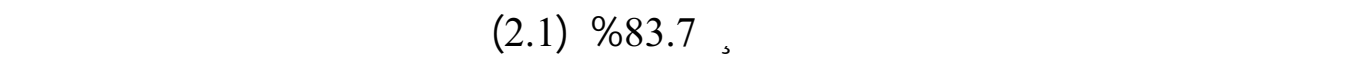

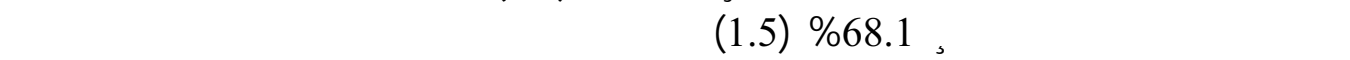

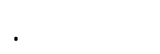

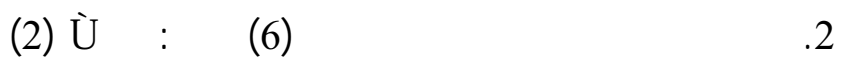

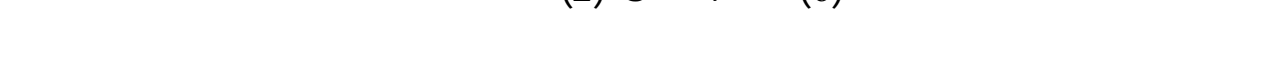

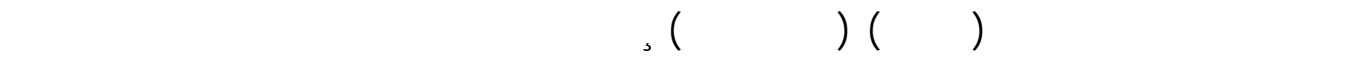

: Ǵó

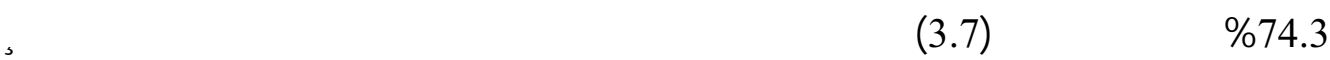

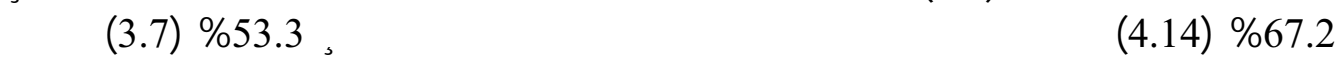

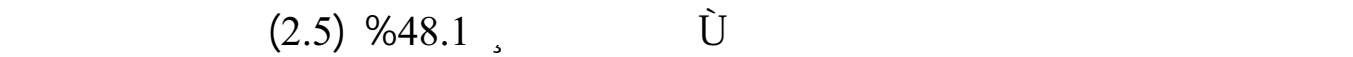

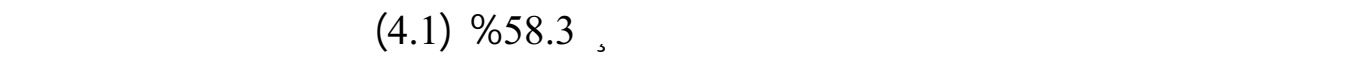
ry

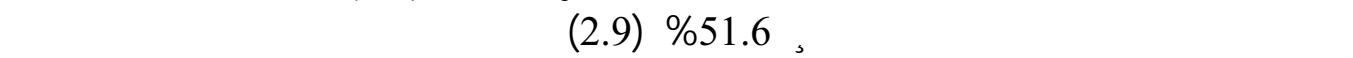

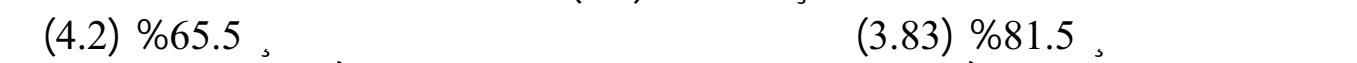

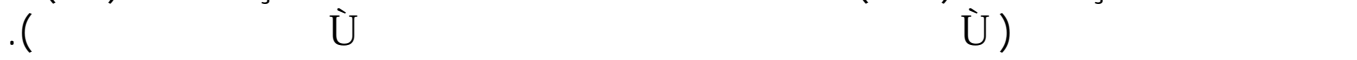

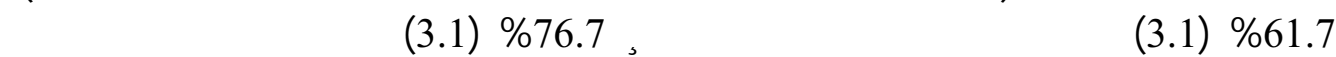

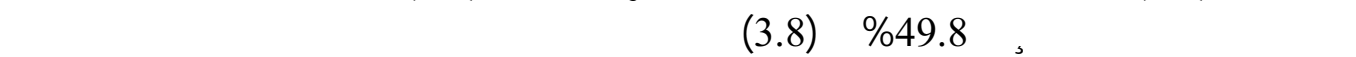

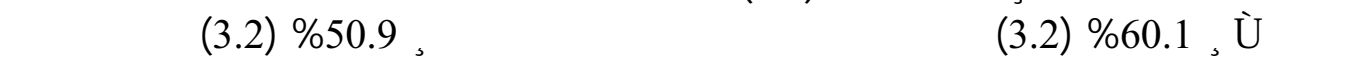

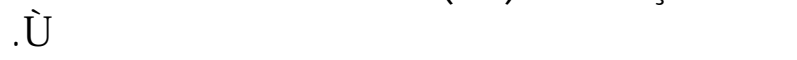

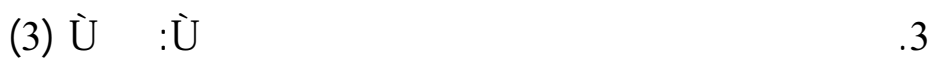

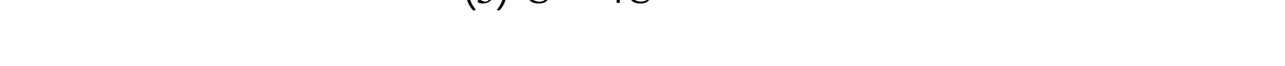

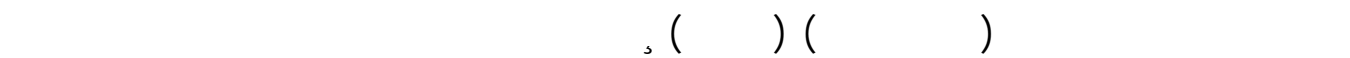

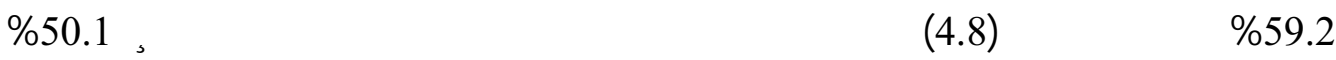

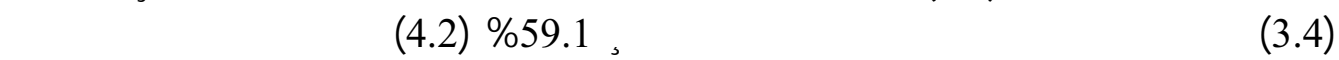

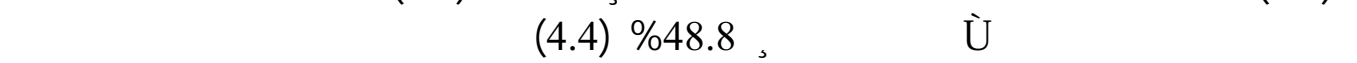

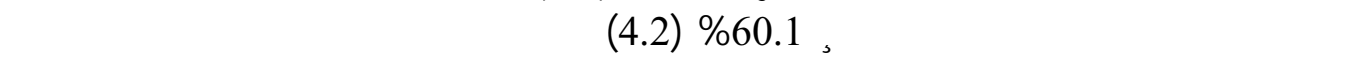

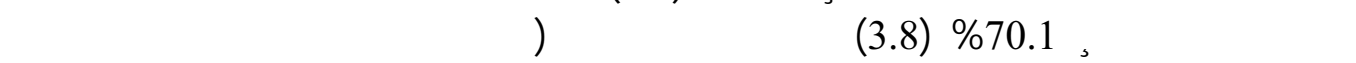

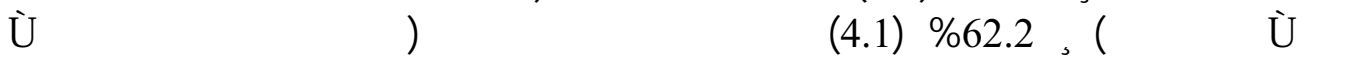

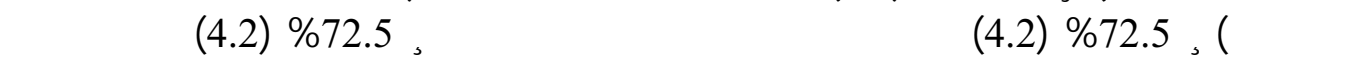

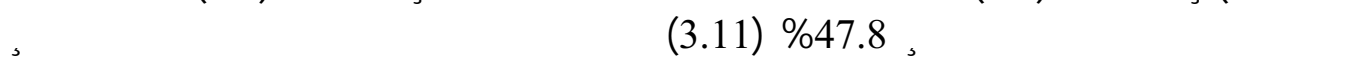

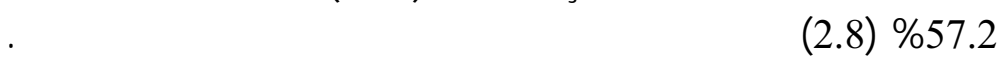

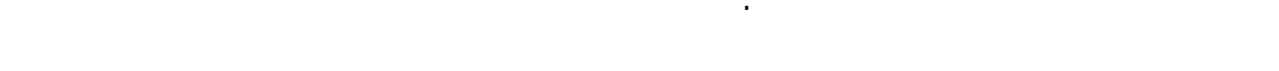

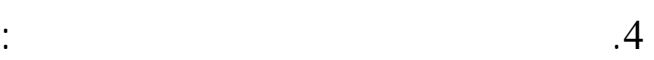

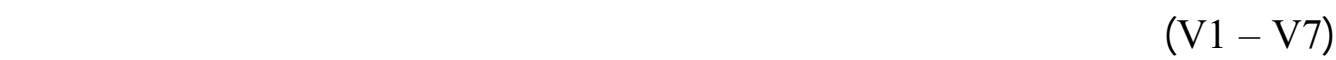

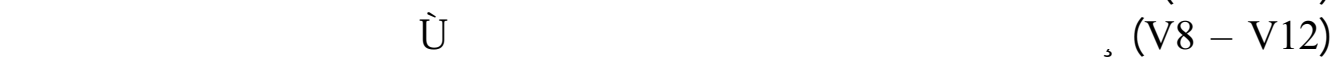

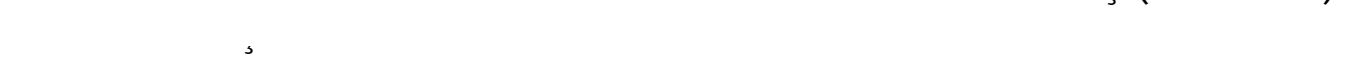

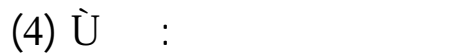

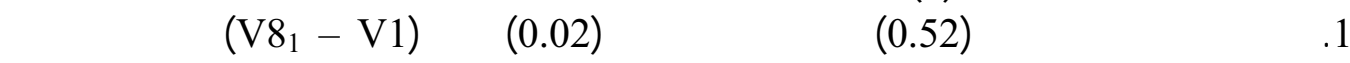

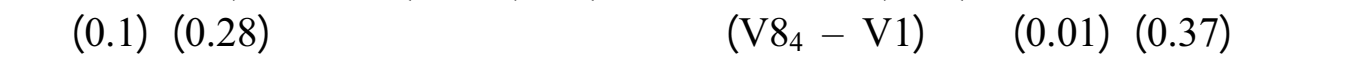




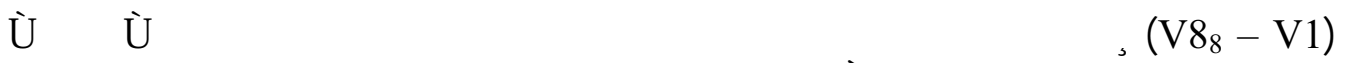

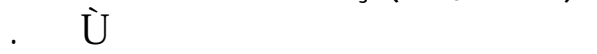

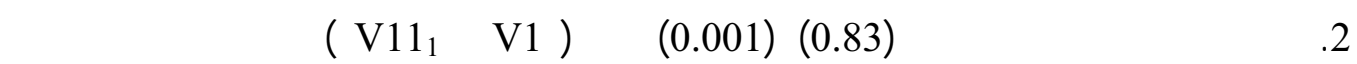

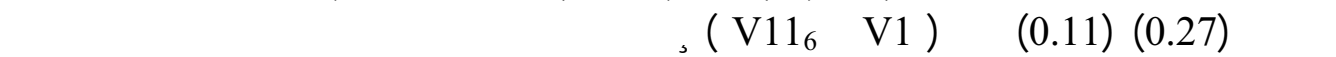

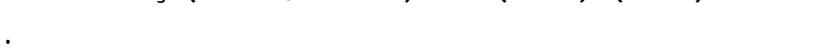

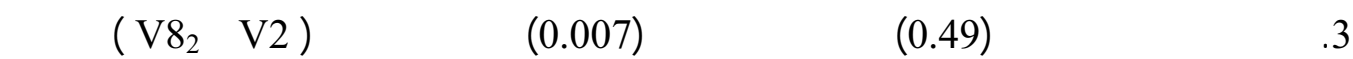
Ś

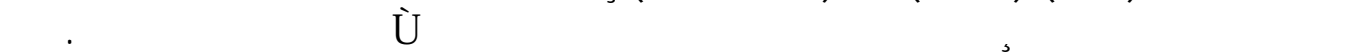

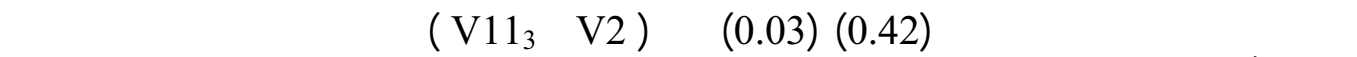
.řgtũ

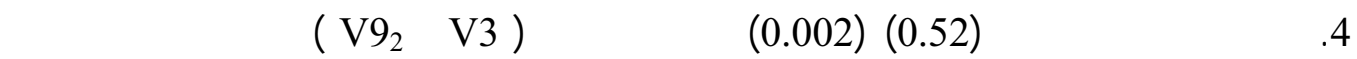

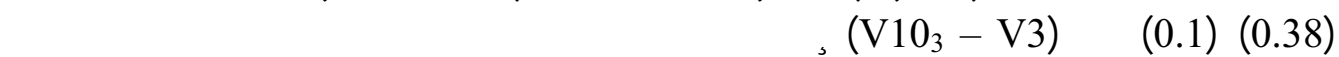

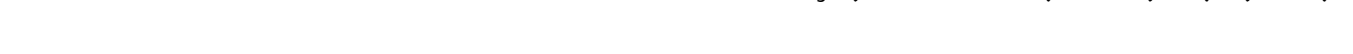

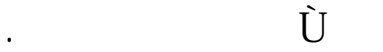

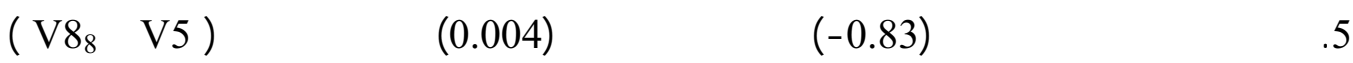

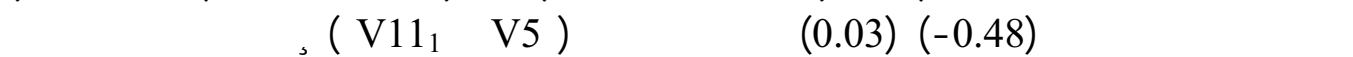

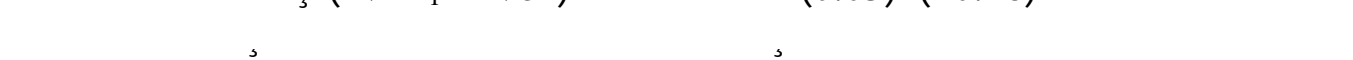

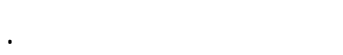

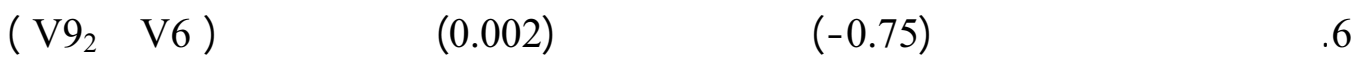

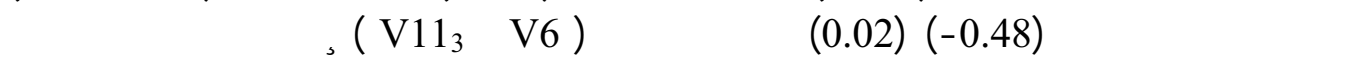

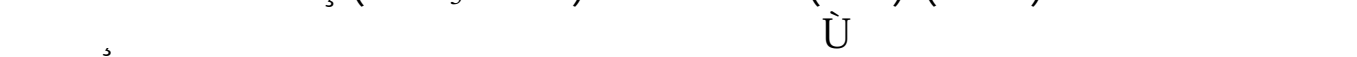

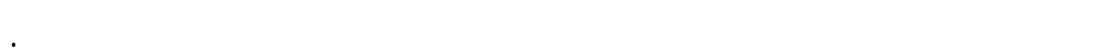

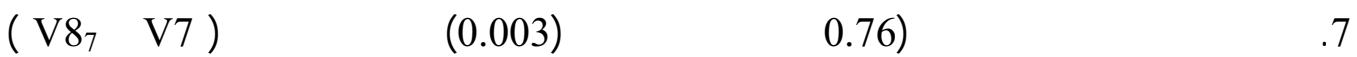

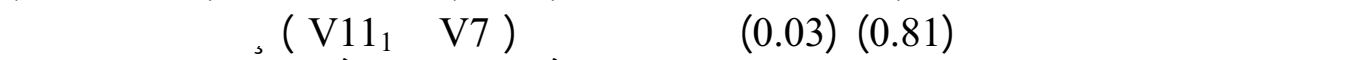

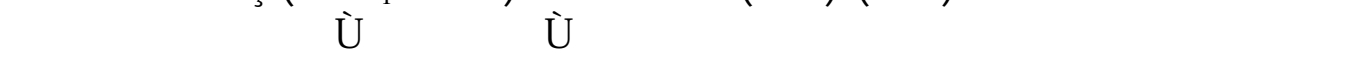

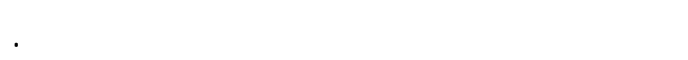

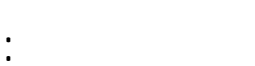

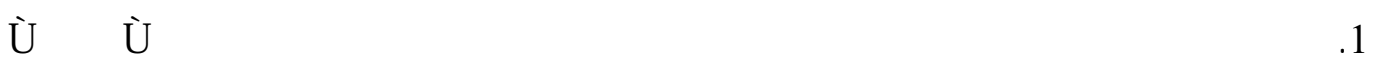

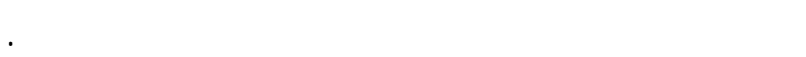

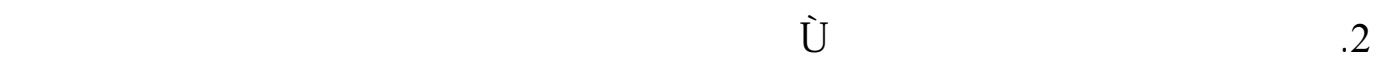

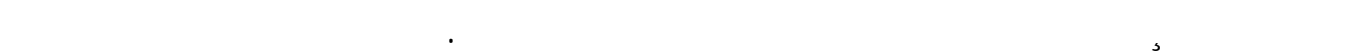

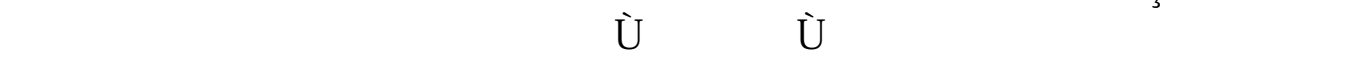

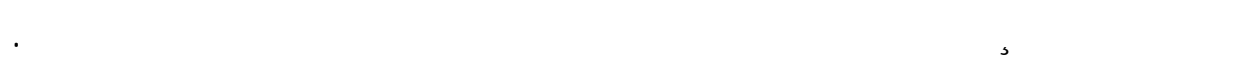

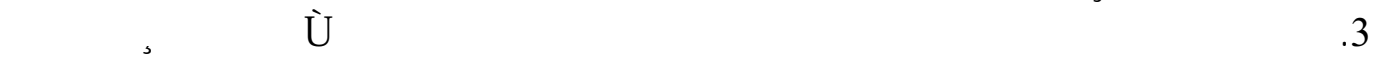

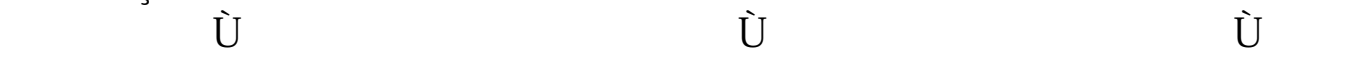

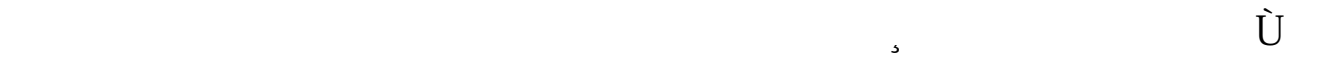

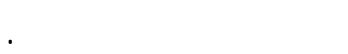

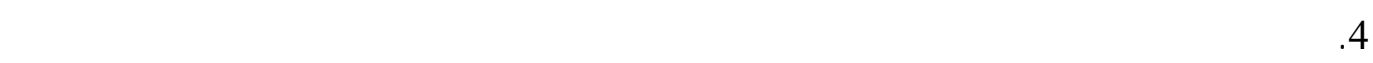

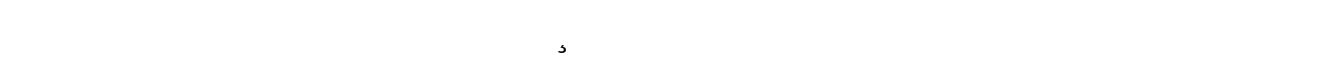

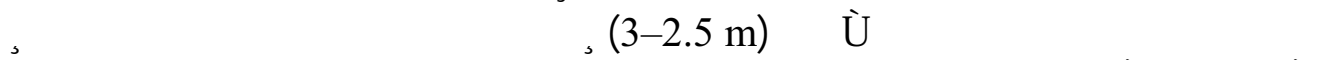

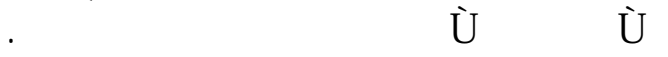




\begin{tabular}{llll} 
Al-Rafidain Engineering & Vol.14 & No.2 & 2006 \\
\hline
\end{tabular}

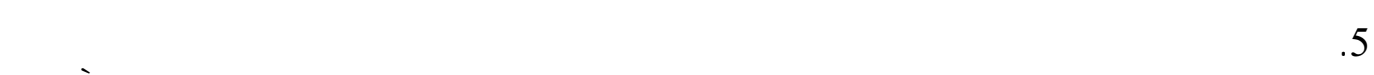

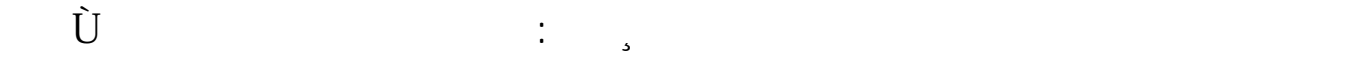

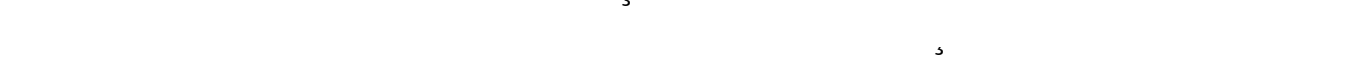

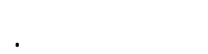

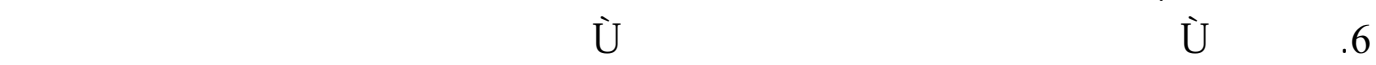

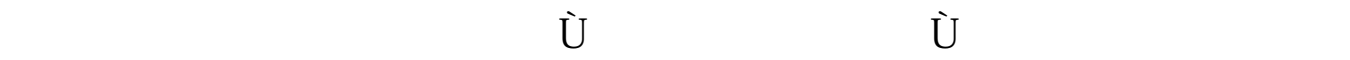

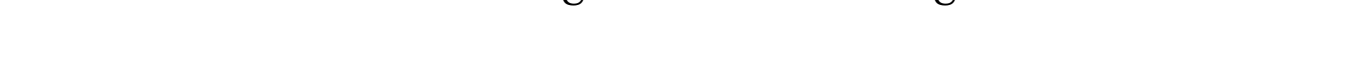

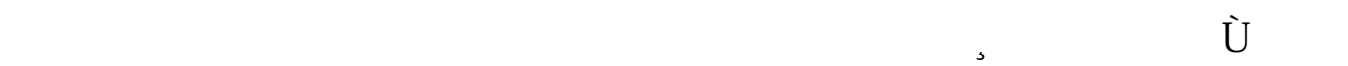

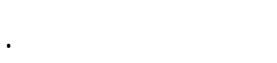

:Ũtứ dŔ

1. Kliment, Stephen.A, "Building Type Basics for Healthcare Facilities", John Wiley \& Sons, Inc/New York, 2000.pp(150-157).

2. Horsburgh, Robert, "Healing by Design" New England Journal of Medicine, September, 14, 1995.

3. Clipson, Colin.W., "Planning for Cardiac Care", University of Michigan, 1973, $\mathrm{pp}(57-60)$.

4. Canter, David, "Designing for therapeutic Environments" John Wiley \& Sone, New York, 1979, pp(190-195), p289, p120.

5. Bednar, Michale J.,"Barrier, Free Environments”, University of Virginia, 1977, $\operatorname{pp}(177-184)$.

6. Lang, Jon, "Creating Architectural Theory" Van Nostrand Relnhold Company, New York, 1987, pp(145-155).

7. Lang, Jon, "Designing for Human Behavior" University of Pennsylvania, 1974, pp(60-71).

8. Staker, David, "Privacy and Dignity", Macclesfield District General Hospital, Patient Environment Assessment Teams, January_May. 2003, Peter.Williamson@Echeshire-tr.nwest.nhs.uk.

9. Hill Country Memorial Health System, "Patient Privacy Policy" Fredericksbury, Texas, December 9, 1998.

10. Shoshkes, Lila, "Space Planing", Architectural Record Books, New York, 1976, pp(103-106). 


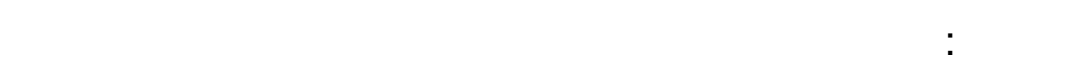

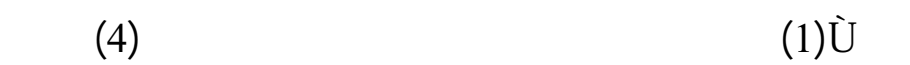

\begin{tabular}{|c|c|c|c|c|c|c|c|c|c|c|}
\hline \multicolumn{2}{|c|}{ P̌ự brśôt } & \multicolumn{2}{|c|}{ brś̂́t } & \multicolumn{2}{|c|}{ ŕctţ bbfśō } & \multicolumn{2}{|c|}{ brśō } & \multicolumn{2}{|c|}{ P̌ṭ̛̛̣ biśō } & \multirow{2}{*}{ ũguśśtí } \\
\hline 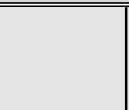 & $\%$ & 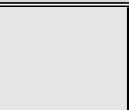 & $\%$ & 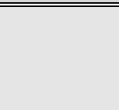 & $\%$ & 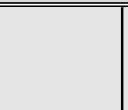 & $\%$ & 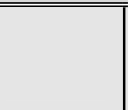 & $\%$ & \\
\hline & & & & 2.72 & 30.69 & & & & & $\mathrm{~V} 8_{1}$ \\
\hline & & 3.55 & 60.92 & & & & & & & $\mathrm{~V} 8_{2}$ \\
\hline & & & & & & 2.42 & 41.85 & & & $\mathrm{~V}_{3}$ \\
\hline 2.71 & 32.31 & & & & & & & & & $\mathrm{~V}_{4}$ \\
\hline & & & & & & 2.67 & 39.82 & & & $\mathrm{~V}_{5}$ \\
\hline & & & & & & & & 2.73 & 30.91 & $\mathrm{~V} 8_{6}$ \\
\hline & & 3.14 & 50.31 & & & & & & & $\mathrm{~V} 8_{7}$ \\
\hline & & & & 3.81 & 34.14 & & & & & $\mathrm{~V}_{8}$ \\
\hline & & & & & & 1.85 & 41.73 & & & $\mathrm{~V} 8_{9}$ \\
\hline & & & & 2.01 & 46.32 & & & & & $\mathrm{~V} 8_{10}$ \\
\hline & & 3.15 & 48.21 & & & & & & & $\mathrm{~V} 9_{1}$ \\
\hline & & & & 3.42 & 51.14 & & & & & $\mathrm{~V} 9_{2}$ \\
\hline & & & & & & 1.8 & 60.12 & & & $\mathrm{~V} 9_{3}$ \\
\hline & & & & & & 2.21 & 45.34 & & & $\mathrm{~V} 9_{4}$ \\
\hline & & & & 3.28 & 49.31 & & & & & $\mathrm{~V} 9_{5}$ \\
\hline & & 3.57 & 52.12 & & & & & & & $\mathrm{~V} 10_{1}$ \\
\hline & & & & & & 1.7 & 71.42 & & & $\mathrm{~V} 10_{2}$ \\
\hline & & & & 3.43 & 45.16 & & & & & ${\mathrm{~V} 10_{3}}_{3}$ \\
\hline & & & & & & 2.4 & 41.93 & & & $\mathrm{~V} 11_{1}$ \\
\hline & & & & & & 2.2 & 39.14 & & & $\mathrm{~V} 11_{2}$ \\
\hline & & & & & & 2.5 & 43.14 & & & $\mathrm{~V}_{11} 1_{3}$ \\
\hline & & & & & & 1.85 & 85.41 & & & $\mathrm{~V} 11_{4}$ \\
\hline & & & & & & 1.71 & 72.41 & & & $\mathrm{~V} 11_{5}$ \\
\hline & & 3.28 & 31.15 & & & & & & & $\mathrm{~V} 11_{6}$ \\
\hline & & 3.28 & 58.13 & & & & & & & $\mathrm{~V}_{11} 1_{7}$ \\
\hline & & & & & & & & 2.11 & 42.81 & $\mathrm{~V} 11_{8}$ \\
\hline & & & & & & & & 2.25 & 30.16 & V11 9 \\
\hline & & & & & & 2.12 & 83.71 & & & V12 \\
\hline & & & & 2.5 & 57.14 & & & & & $\mathrm{~V} 12_{2}$ \\
\hline & & & & 2.41 & 52.85 & & & & & $\mathrm{~V} 12_{3}$ \\
\hline & & & & & & & & 1.50 & 68.11 & $\mathrm{~V} 12_{4}$ \\
\hline
\end{tabular}




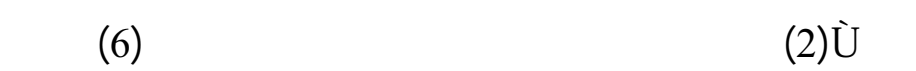

\begin{tabular}{|c|c|c|c|c|c|c|c|c|c|c|}
\hline \multicolumn{2}{|c|}{ Řtự brśỗ } & \multicolumn{2}{|c|}{ brśôt } & \multicolumn{2}{|c|}{ 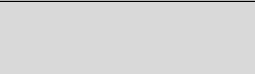 } & \multicolumn{2}{|c|}{ biśō } & \multicolumn{2}{|c|}{ P̌tự biśō } & \multirow{2}{*}{ ũưzśtó } \\
\hline 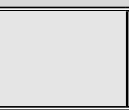 & $\%$ & 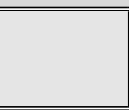 & $\%$ & 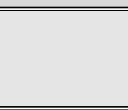 & $\%$ & 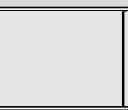 & $\%$ & 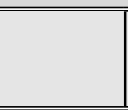 & $\%$ & \\
\hline & & 3.71 & 74.32 & & & & & & & $\mathrm{~V} 8_{1}$ \\
\hline \multirow[t]{5}{*}{4.14} & 67.02 & & & & & & & & & $\mathrm{~V} 8_{2}$ \\
\hline & & & & 2.85 & 32.87 & & & & & $\mathrm{~V}_{3}$ \\
\hline & & & & 2.7 & 53.32 & & & & & $\mathrm{~V} 8_{4}$ \\
\hline & & & & 2.52 & 48.14 & & & & & $\mathrm{~V} 8_{5}$ \\
\hline & & & & 3.00 & 52.31 & & & & & $\mathrm{~V} 8_{6}$ \\
\hline 4.1 & 58.97 & & & & & & & & & $\mathrm{~V} 8_{7}$ \\
\hline \multirow[t]{8}{*}{3.82} & 44.52 & & & & & & & & & $\mathrm{~V} 8_{8}$ \\
\hline & & & & & & 2.28 & 68.72 & & & $\mathrm{~V}_{9}$ \\
\hline & & & & 2.42 & 51.14 & & & & & $\mathrm{~V} 8_{10}$ \\
\hline & & 3.12 & 31.32 & & & & & & & $\mathrm{~V} 9_{1}$ \\
\hline & & 2.91 & 51.67 & & & & & & & $\mathrm{~V} 9_{2}$ \\
\hline & & & & & & 2.10 & 71.42 & & & $\mathrm{~V} 9_{3}$ \\
\hline & & & & & & 2.28 & 69.31 & & & $\mathrm{~V} 9_{4}$ \\
\hline & & 3.83 & 81.52 & & & & & & & $\mathrm{~V} 9_{5}$ \\
\hline \multirow[t]{16}{*}{4.2} & 65.5 & & & & & & & & & $\mathrm{~V} 10_{1}$ \\
\hline & & & & & & 2.14 & 51.31 & & & $\mathrm{~V} 10_{2}$ \\
\hline & & & & 2.81 & 38.92 & & & & & $\mathrm{~V} 10_{3}$ \\
\hline & & 3.11 & 61.72 & & & & & & & V11 \\
\hline & & 2.71 & 76.42 & & & & & & & $\mathrm{~V} 11_{2}$ \\
\hline & & 3.84 & 49.81 & & & & & & & $\mathrm{~V} 11_{3}$ \\
\hline & & & & 2.28 & 28.5 & 2.28 & 28.5 & & & V11 \\
\hline & & & & & & 2.01 & 70.14 & & & $\mathrm{~V} 11_{5}$ \\
\hline & & 3.2 & 60.12 & & & & & & & V11 6 \\
\hline & & & & 2.81 & 48.82 & & & & & V11 \\
\hline & & & & & & 2.61 & 62.78 & & & $\mathrm{~V} 11_{8}$ \\
\hline & & & & 2.4 & 47.1 & & & & & V11 9 \\
\hline & & & & & & 2.51 & 39.92 & & & V12 \\
\hline & & & & 3.42 & 42.85 & & & & & $\mathrm{~V} 12_{2}$ \\
\hline & & & & 3.11 & 50.9 & & & & & V123 \\
\hline & & & & & & 2.29 & 60.91 & & & V12 \\
\hline
\end{tabular}




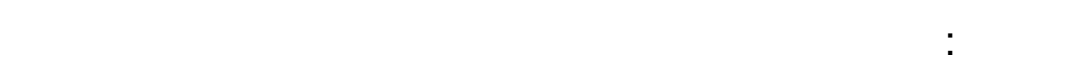

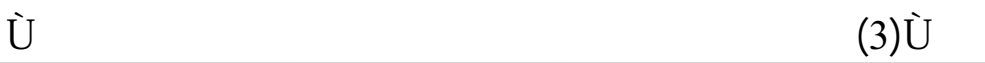

\begin{tabular}{|c|c|c|c|c|c|c|c|c|c|c|}
\hline \multicolumn{2}{|c|}{ Řtự brśôt } & \multicolumn{2}{|c|}{ brśôt } & \multicolumn{2}{|c|}{ 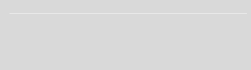 } & \multicolumn{2}{|c|}{ brśō } & \multicolumn{2}{|c|}{ P̌tự biśó } & \multirow{2}{*}{ ũzzśśd } \\
\hline 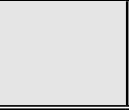 & $\%$ & 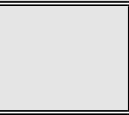 & $\%$ & 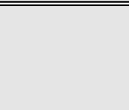 & $\%$ & 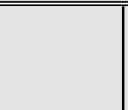 & $\%$ & 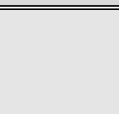 & $\%$ & \\
\hline & & 3.11 & 43.2 & & & & & & & $\mathrm{~V} 8_{1}$ \\
\hline \multirow[t]{3}{*}{4.8} & 59.28 & & & & & & & & & $\mathrm{~V} 8_{2}$ \\
\hline & & 3.4 & 50.19 & & & & & & & $\mathrm{~V}_{3}$ \\
\hline & & 4.21 & 59.14 & & & & & & & $\mathrm{~V}_{4}$ \\
\hline \multirow[t]{2}{*}{4.41} & 48.8 & & & & & & & & & $\mathrm{~V} 8_{5}$ \\
\hline & & & & 3.1 & 42.86 & & & & & $\mathrm{~V}_{6}$ \\
\hline 4.31 & 49.81 & & & & & & & & & $\mathrm{~V} 8_{7}$ \\
\hline \multirow[t]{7}{*}{4.21} & 60.13 & & & & & & & & & $\mathrm{~V}_{8}$ \\
\hline & & & & & & 2.12 & 38.81 & & & V8 9 \\
\hline & & & & & & 1.81 & 42.86 & & & $\mathrm{~V} 8_{10}$ \\
\hline & & & & & & 2.80 & 57.14 & & & $\mathrm{~V} 9_{1}$ \\
\hline & & 3.5 & 43.12 & & & & & & & $\mathrm{~V} 9_{2}$ \\
\hline & & & & 2.12 & 44.21 & & & & & $\mathrm{~V} 9_{3}$ \\
\hline & & & & & & 2.41 & 39.14 & & & $\mathrm{~V} 9_{4}$ \\
\hline \multirow[t]{3}{*}{4.22} & 47.71 & & & & & & & & & $\mathrm{~V} 9_{5}$ \\
\hline & & 3.8 & 70.14 & & & & & & & $\mathrm{~V} 10_{1}$ \\
\hline & & & & & & 2.7 & 42.18 & & & $\mathrm{~V} 10_{2}$ \\
\hline 4.1 & 62.18 & & & & & & & & & ${\mathrm{~V} 10_{3}}$ \\
\hline \multirow[t]{5}{*}{4.2} & 72.51 & & & & & & & & & V11 \\
\hline & & & & 3.22 & 58.21 & & & & & $\mathrm{~V} 11_{2}$ \\
\hline & & 4.2 & 72.55 & & & & & & & $\mathrm{~V} 11_{3}$ \\
\hline & & & & & & 2.42 & 51.89 & & & $\mathrm{~V} 11_{4}$ \\
\hline & & & & & & & & 2.23 & 42.1 & $\mathrm{~V} 11_{5}$ \\
\hline \multirow[t]{8}{*}{3.11} & 47.8 & & & & & & & & & V11 6 \\
\hline & & 3.91 & 49.21 & & & & & & & ${\mathrm{~V} 11_{7}}_{4}$ \\
\hline & & & & & & & & 2.51 & 28.5 & $\mathrm{~V} 11_{8}$ \\
\hline & & & & & & 2.61 & 31.32 & & & V11 ${ }_{9}$ \\
\hline & & & & & & & & 2.55 & 42.11 & $\mathrm{~V} 12_{1}$ \\
\hline & & & & 2.8 & 57.24 & & & & & $\mathrm{~V} 12_{2}$ \\
\hline & & & & 2.4 & 42.14 & & & & & $\mathrm{~V} 12_{3}$ \\
\hline & & & & & & & & 1.44 & 75.39 & V12 \\
\hline
\end{tabular}




\begin{tabular}{llll} 
Al-Rafidain Engineering & Vol.14 & No.2 & 2006 \\
\hline
\end{tabular}

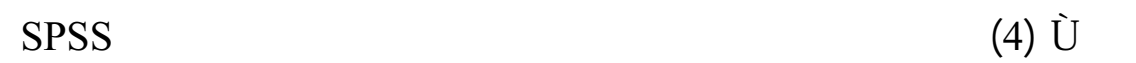

\begin{tabular}{|c|c|c|c|c|c|c|c|c|c|c|c|c|c|c|}
\hline \multicolumn{2}{|c|}{ V7 } & \multicolumn{2}{|c|}{ V6 } & \multicolumn{2}{|c|}{ V5 } & \multicolumn{2}{|c|}{ V4 } & \multicolumn{2}{|c|}{ V3 } & \multicolumn{2}{|c|}{ V2 } & \multicolumn{2}{|c|}{ V1 } & \multirow{2}{*}{ 感 } \\
\hline $\mathrm{r}$ & 頮: & $\mathrm{r}$ & 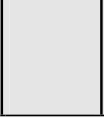 & $r$ & 资 & $\mathrm{r}$ & 畓 & $\mathrm{r}$ & 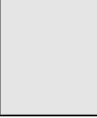 & $\mathrm{r}$ & 褚: & $r$ & 䙓 & \\
\hline & & & & 0.18 & 0.7 & 0.13 & 0.3 & 0.18 & 0.02 & & & 0.52 & 0.02 & $\mathrm{~V} 8_{1}$ \\
\hline \multirow[t]{5}{*}{0.11} & 0.02 & & & & & & & & & 0.49 & .007 & & & $\mathrm{~V}_{2}$ \\
\hline & & & & & & & & & & & & & & $\mathrm{V}_{3}$ \\
\hline & & & & & & & & & & 0.54 & .003 & 0.37 & 0.01 & $\mathrm{~V}_{4}$ \\
\hline & & & & & & & & -0.19 & 0.2 & & & & & $\mathrm{~V} 8_{5}$ \\
\hline & & & & & & -0.16 & 0.5 & & & & & 0.11 & 0.2 & $\mathrm{~V}_{6}$ \\
\hline \multirow[t]{3}{*}{0.76} & .003 & & & & & & & & & & & & & $\mathrm{~V} 8_{7}$ \\
\hline & & & & -0.83 & .004 & & & & & & & 0.28 & 0.1 & $\mathrm{~V}_{8}$ \\
\hline & & & & & & & & & & & & & & $\mathrm{V}_{9}$ \\
\hline \multirow[t]{9}{*}{-0.21} & 0.5 & & & & & & & & & -0.13 & 0.1 & & & $\mathrm{~V} 8_{10}$ \\
\hline & & & & & & & & & & & & & & $\mathrm{V} 9_{1}$ \\
\hline & & -0.75 & .002 & & & & & 0.52 & .002 & & & -0.21 & 0.3 & $\mathrm{~V} 9_{2}$ \\
\hline & & & & -0.19 & 0.8 & & & & & & & & & $\mathrm{~V} 9_{3}$ \\
\hline & & & & & & & & & & & & & & $\mathrm{V} 9_{4}$ \\
\hline & & & & & & 0.22 & 0.7 & & & & & & & $\mathrm{~V} 9_{5}$ \\
\hline & & & & & & & & & & -0.25 & 0.6 & & & $\mathrm{~V} 10_{1}$ \\
\hline & & & & & & & & & & & & -0.12 & 0.4 & $\mathrm{~V} 10_{2}$ \\
\hline & & -0.48 & 0.02 & & & & & 0.38 & 0.1 & & & & & $\mathrm{~V} 10_{3}$ \\
\hline \multirow[t]{7}{*}{0.81} & 0.03 & & & -0.48 & 0.03 & & & & & & & 0.83 & .001 & $\mathrm{~V} 11_{1}$ \\
\hline & & & & & & & & & & & & & & $\mathrm{V} 11_{2}$ \\
\hline & & & & & & -0.12 & 0.3 & & & 0.42 & 0.03 & & & $\mathrm{~V} 11_{3}$ \\
\hline & & & & & & & & & & & & & & $\mathrm{V} 11_{4}$ \\
\hline & & 0.24 & 0.9 & & & & & 0.22 & 0.3 & & & & & $\mathrm{~V} 11_{5}$ \\
\hline & & & & & & & & & & & & 0.27 & 0.11 & $\mathrm{~V} 11_{6}$ \\
\hline & & & & & & & & & & & & & & $\mathrm{V} 11_{7}$ \\
\hline \multirow[t]{3}{*}{0.17} & 0.9 & & & & & & & & & 0.17 & 0.3 & & & $\mathrm{~V} 11_{8}$ \\
\hline & & & & -0.11 & 0.6 & & & & & & & & & V11 \\
\hline & & & & & & & & -0.15 & 0.5 & & & 0.17 & 0.5 & $\mathrm{~V} 12_{1}$ \\
\hline \multirow[t]{3}{*}{-0.14} & 0.4 & & & & & & & & & & & & & $\mathrm{~V} 12_{2}$ \\
\hline & & & & & & 0.17 & 0.01 & & & & & & & $\mathrm{~V} 12_{3}$ \\
\hline & & & & & & & & & & & & 0.21 & 0.5 & V12 \\
\hline
\end{tabular}




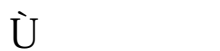

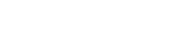

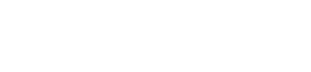

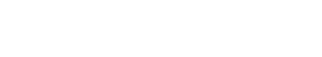

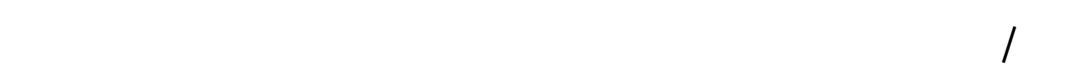

:rv̂tutur

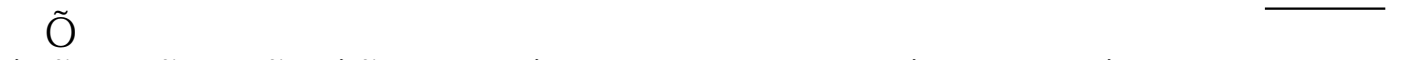

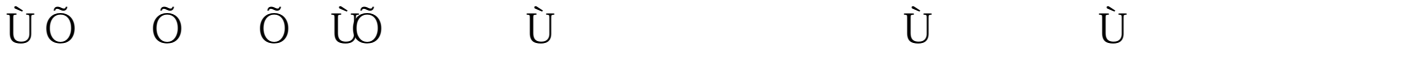

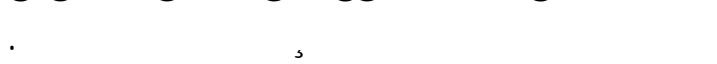

60 Fl̆41 Đr $\square$

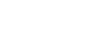

GÝ̛̛́S

:řtadŔ

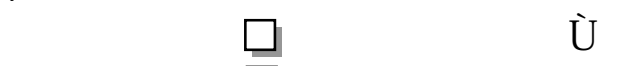

Ř̃ugr

F̆

řŭesé

rựús

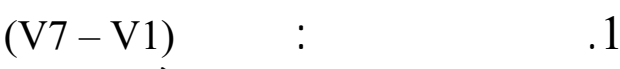
$20 Đ C$ ¿'̄

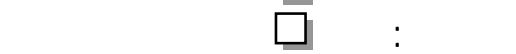
:ŨC̛̈ß́

Gdóǵń

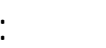




\begin{tabular}{llll} 
Al-Rafidain Engineering & Vol.14 & No.2 & 2006 \\
\hline
\end{tabular}

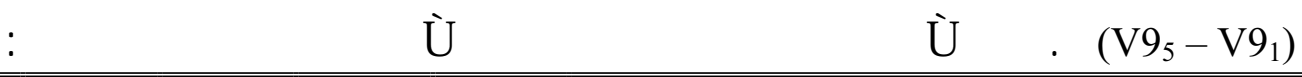

\begin{tabular}{|c|c|c|c|c|c|}
\hline $\begin{array}{c}\text { bf'sot } \\
\text { P̌tur }\end{array}$ & brśôx & 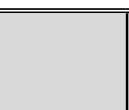 & bfśŌ & 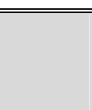 & 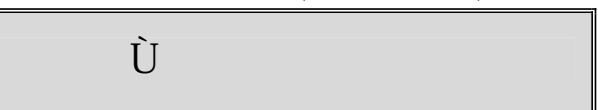 \\
\hline & & & & & 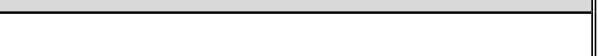 \\
\hline & & & & & 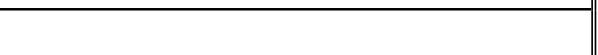 \\
\hline & & & & & '万 \\
\hline & & & & & 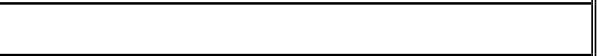 \\
\hline & & & & & 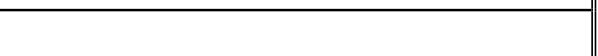 \\
\hline
\end{tabular}

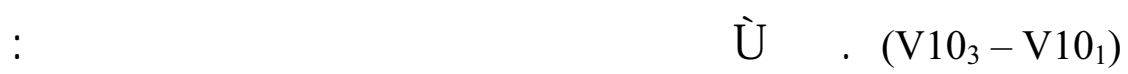

\begin{tabular}{|c|c|c|c|c|c|}
\hline 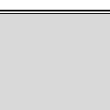 & biśôt & 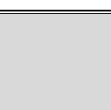 & ЂfśŌ & 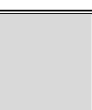 & ¿व̈̈̈̈Ŕ \\
\hline & & & & & 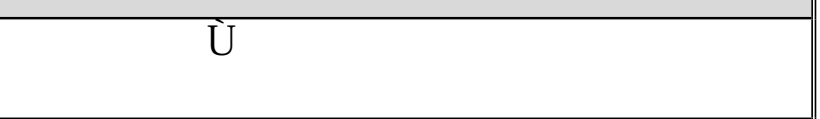 \\
\hline & & & & & 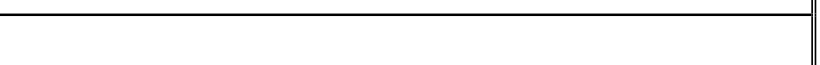 \\
\hline & & & & & 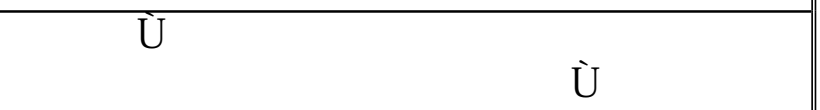 \\
\hline
\end{tabular}

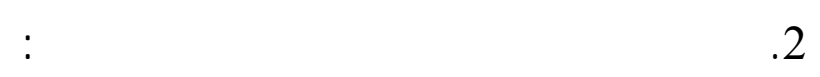

(V11 ${ }_{9}$ - :b»

V11 1 )

\begin{tabular}{|c|c|c|c|c|c|}
\hline 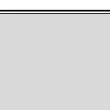 & biśôt & 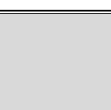 & ЂfśŌ & 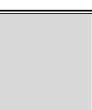 & 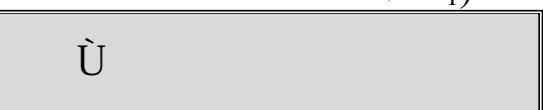 \\
\hline & & & & & 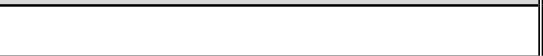 \\
\hline & & & & & 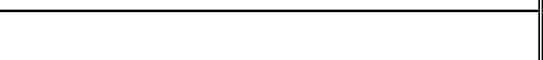 \\
\hline & & & & & 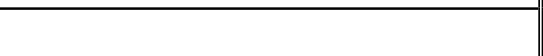 \\
\hline & & & & & 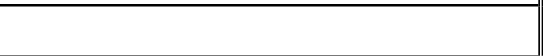 \\
\hline & & & & & Sróćt \\
\hline & & & & & 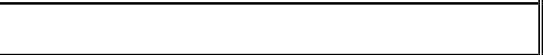 \\
\hline & & & & & 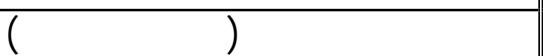 \\
\hline & & & & & 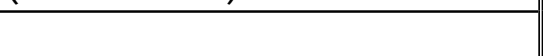 \\
\hline & & & & & 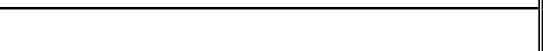 \\
\hline
\end{tabular}

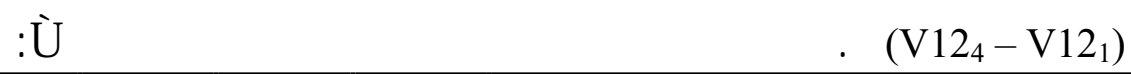

\begin{tabular}{|c|c|c|c|c|c|}
\hline $\begin{array}{c}\text { biśót } \\
\text { P̌tur }\end{array}$ & Ђfśôt & 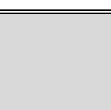 & brśō & 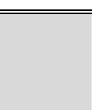 & ¿वOARR \\
\hline & & & & & 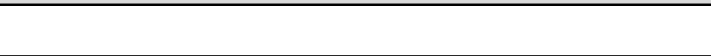 \\
\hline & & & & & 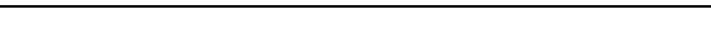 \\
\hline & & & & & 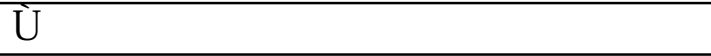 \\
\hline & & & & & 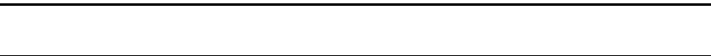 \\
\hline
\end{tabular}

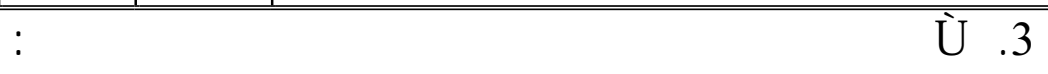

